MATHEMATICS OF COMPUTATION

Volume 76, Number 258, April 2007, Pages 573-595

S $0025-5718(06) 01950-8$

Article electronically published on December 27, 2006

\title{
A LOCALLY DIVERGENCE-FREE NONCONFORMING FINITE ELEMENT METHOD FOR THE TIME-HARMONIC MAXWELL EQUATIONS
}

\author{
SUSANNE C. BRENNER, FENGYAN LI, AND LI-YENG SUNG
}

\begin{abstract}
A new numerical method for computing the divergence-free part of the solution of the time-harmonic Maxwell equations is studied in this paper. It is based on a discretization that uses the locally divergence-free CrouzeixRaviart nonconforming $P_{1}$ vector fields and includes a consistency term involving the jumps of the vector fields across element boundaries. Optimal convergence rates (up to an arbitrary positive $\epsilon$ ) in both the energy norm and the $L_{2}$ norm are established on graded meshes. The theoretical results are confirmed by numerical experiments.
\end{abstract}

\section{INTRODUCTION}

Let $\Omega \subset \mathbb{R}^{2}$ be a bounded polygonal domain, $\boldsymbol{f} \in\left[L_{2}(\Omega)\right]^{2}$ and $k \geq 0$. Consider the variational problem of the time-harmonic Maxwell equations with the perfectly conducting boundary condition:

Find $\boldsymbol{u} \in H_{0}(\operatorname{curl} ; \Omega)$ such that

$$
(\nabla \times \boldsymbol{u}, \nabla \times \boldsymbol{v})-k^{2}(\boldsymbol{u}, \boldsymbol{v})=(\boldsymbol{f}, \boldsymbol{v}) \quad \forall \boldsymbol{v} \in H_{0}(\operatorname{curl} ; \Omega),
$$

where $(\cdot, \cdot)$ denotes the inner product of $L_{2}(\Omega)$ (or $\left.\left[L_{2}(\Omega)\right]^{2}\right)$,

$$
H(\operatorname{curl} ; \Omega)=\left\{\boldsymbol{v}=\left[\begin{array}{l}
v_{1} \\
v_{2}
\end{array}\right] \in\left[L_{2}(\Omega)\right]^{2}: \nabla \times \boldsymbol{v}=\frac{\partial v_{2}}{\partial x_{1}}-\frac{\partial v_{1}}{\partial x_{2}} \in L_{2}(\Omega)\right\}
$$

and

$$
H_{0}(\operatorname{curl} ; \Omega)=\{\boldsymbol{v} \in H(\operatorname{curl} ; \Omega): \boldsymbol{n} \times \boldsymbol{v}=0 \text { on } \partial \Omega\} .
$$

Here the vector $\boldsymbol{n}$ denotes the unit outer normal on $\partial \Omega$. We assume that $k^{2}$ is not one of the Maxwell eigenvalues and hence (1.1) has a unique solution in $H_{0}(\operatorname{curl} ; \Omega)$.

The space $H_{0}(\operatorname{curl} ; \Omega)$ admits the well-known Helmholtz-Hodge decomposition [12, 15

$$
H_{0}(\operatorname{curl} ; \Omega)=\left[H_{0}(\operatorname{curl} ; \Omega) \cap H\left(\operatorname{div}^{0} ; \Omega\right)\right] \oplus \nabla H_{0}^{1}(\Omega),
$$

Received by the editor August 8, 2005 and, in revised form, April 19, 2006.

2000 Mathematics Subject Classification. Primary 65N30, 65N15, 35Q60.

Key words and phrases. Time-harmonic Maxwell equations, nonconforming finite element methods.

The work of the first author was supported in part by the National Science Foundation under Grant No. DMS-03-11790. 
where

$$
H\left(\operatorname{div}^{0} ; \Omega\right)=\left\{\boldsymbol{v}=\left[\begin{array}{l}
v_{1} \\
v_{2}
\end{array}\right] \in\left[L_{2}(\Omega)\right]^{2}: \nabla \cdot \boldsymbol{v}=\frac{\partial v_{1}}{\partial x_{1}}+\frac{\partial v_{2}}{\partial x_{2}}=0\right\},
$$

and the direct sum is orthogonal with respect to the inner product of $\left[L_{2}(\Omega)\right]^{2}$.

According to (1.2), we can write

$$
\boldsymbol{u}=\stackrel{\circ}{\boldsymbol{u}}+\nabla \phi
$$

where $\stackrel{\circ}{\boldsymbol{u}} \in H_{0}(\operatorname{curl} ; \Omega) \cap H\left(\operatorname{div}^{0} ; \Omega\right)$ and $\phi \in H_{0}^{1}(\Omega)$. From (1.1) we see that $\stackrel{\circ}{\boldsymbol{u}}$ and $\phi$ satisfy the following equations:

$$
(\nabla \times \stackrel{\circ}{\boldsymbol{u}}, \nabla \times \boldsymbol{v})-k^{2}(\stackrel{\circ}{\boldsymbol{u}}, \boldsymbol{v})=(\boldsymbol{f}, \boldsymbol{v})
$$

for all $\boldsymbol{v} \in H_{0}(\operatorname{curl} ; \Omega) \cap H\left(\operatorname{div}^{0} ; \Omega\right)$,

$$
-k^{2}(\nabla \phi, \nabla \psi)=(\boldsymbol{f}, \nabla \psi)
$$

for all $\psi \in H_{0}^{1}(\Omega)$.

Since $\phi$ can be obtained from the Poisson equation (1.4), we will focus on (1.3), which will be referred to as the reduced time-harmonic Maxwell (RTHM) equations. Under the assumption that $k^{2}$ is not a Maxwell eigenvalue, the RTHM equations have a unique solution in $H_{0}(\operatorname{curl} ; \Omega) \cap H\left(\operatorname{div}^{0} ; \Omega\right)$. Note that the strong form of the RTHM equations is given by

$$
\nabla \times(\nabla \times \stackrel{\circ}{\boldsymbol{u}})-k^{2} \stackrel{\circ}{\boldsymbol{u}}=Q \boldsymbol{f},
$$

where $Q$ is the orthogonal projection operator from $\left[L_{2}(\Omega)\right]^{2}$ onto the space $H\left(\operatorname{div}^{0} ; \Omega\right)$. In particular, (1.5) implies that the scalar function $\nabla \times \stackrel{\circ}{\boldsymbol{u}} \in H^{1}(\Omega)$ and

$$
\|\nabla \times \stackrel{\circ}{\boldsymbol{u}}\|_{H^{1}(\Omega)} \leq C_{\Omega, k}\|\boldsymbol{f}\|_{L_{2}(\Omega)}
$$

Remark 1.1. The assumption that $\boldsymbol{f} \in\left[L_{2}(\Omega)\right]^{2}$ is weaker than the assumption $\boldsymbol{f} \in$ $H(\operatorname{div} ; \Omega)$ required for numerical schemes for the time-harmonic Maxwell equations.

In this paper we introduce a numerical method for the RTHM equations using locally divergence-free Crouzeix-Raviart nonconforming $P_{1}$ vector fields [9]. Note that a straightforward discretization of (1.1) using such nonconforming vector fields does not converge (cf. [15], page 200 and Table 7.2 below). Therefore a consistency term involving the jumps of the nonconforming vector fields across interelement boundaries is included in our discretization. We will show that the order of convergence of our method is optimal (up to an arbitrarily small $\epsilon$ ) in both the energy norm and the $L_{2}$ norm, provided properly graded meshes are used. In the spirit of [8], one can say that the results of this paper rehabilitate nonconforming nodal finite elements for the Maxwell equations.

The rest of the paper is organized as follows. We introduce the space of locally divergence-free nonconforming $P_{1}$ vector fields in Section 2 , together with a description of the graded meshes necessary for recovering the optimal convergence rates. The discretization of the RTHM equations is given in Section 3, where an abstract discretization error estimate is also derived. The choice of the grading parameters and the convergence analysis of our method on graded meshes depend on the nature of the singularities at the corners of $\Omega$, which is discussed in Section 4. Section 5 contains four preliminary estimates for the convergence analysis, 
which is carried out in Section 6. Results of numerical experiments are reported in Section 7 , followed by some concluding remarks in Section 8 ,

\section{LOCALLY DIVERGENCE-FREE VECTOR FIELDS ON GRADED MESHES}

Let $\mathcal{T}_{h}$ be a family of triangulations of $\Omega$. We define the space $V_{h}$ of locally divergence-free Crouzeix-Raviart nonconforming $P_{1}$ vector fields 9 by

$$
V_{h}=\left\{\boldsymbol{v} \in\left[L_{2}(\Omega)\right]^{2}: \boldsymbol{v}_{T}=\left.\boldsymbol{v}\right|_{T} \in\left[P_{1}(T)\right]^{2} \text { and } \nabla \cdot \boldsymbol{v}_{T}=0 \forall T \in \mathcal{T}_{h},\right.
$$

$\boldsymbol{v}$ is continuous at the midpoints of the interior edges of $\mathcal{T}_{h}$

and $\boldsymbol{n} \times \boldsymbol{v}=0$ at the midpoints of the edges of $\mathcal{T}_{h}$ along $\left.\partial \Omega\right\}$.

Remark 2.1. For a simply connected $\Omega$, there exists a completely local basis for $V_{h}$ consisting of vector fields tangential to the edges of $\mathcal{T}_{h}$ and vector fields representing rotations around the vertices of $\mathcal{T}_{h}[18,10$. For a multiply connected domain, the basis of $V_{h}$ involves vector fields along cuts that reduce $\Omega$ to a simply connected domain, in addition to local vector fields. The dimension of $V_{h}$ is $\approx(4 / 3) \times$ (the number of edges in $\mathcal{T}_{h}$ ) for a general polygonal domain $\Omega$.

For any $s>\frac{1}{2}$ there is a natural weak interpolation operator $\Pi_{T}:\left[H^{s}(T)\right]^{2} \longrightarrow$ $\left[P_{1}(T)\right]^{2}$ defined by

$$
\left(\Pi_{T} \boldsymbol{\zeta}\right)\left(m_{e_{i}}\right)=\frac{1}{\left|e_{i}\right|} \int_{e_{i}} \boldsymbol{\zeta} d s \quad \text { for } \quad i=1,2,3,
$$

where $m_{e_{i}}$ is the midpoint of the edge $e_{i}$ of $T$. It follows immediately from (2.1) and Green's theorem that

$$
\begin{aligned}
\int_{T} \nabla \times\left(\Pi_{T} \boldsymbol{\zeta}\right) d x & =\int_{T} \nabla \times \boldsymbol{\zeta} d x & & \forall \boldsymbol{\zeta} \in\left[H^{s}(T)\right]^{2}, \\
\int_{T} \nabla \cdot\left(\Pi_{T} \boldsymbol{\zeta}\right) d x & =\int_{T} \nabla \cdot \boldsymbol{\zeta} d x & & \forall \boldsymbol{\zeta} \in\left[H^{s}(T)\right]^{2} .
\end{aligned}
$$

Furthermore, given $s \in(1 / 2,2]$, we have the following interpolation error estimates [9]:

$$
\left\|\boldsymbol{\zeta}-\Pi_{T} \boldsymbol{\zeta}\right\|_{L_{2}(T)}+h_{T}^{\min (s, 1)}\left|\boldsymbol{\zeta}-\Pi_{T} \boldsymbol{\zeta}\right|_{H^{\min (s, 1)}(T)} \leq C_{T} h_{T}^{s}|\boldsymbol{\zeta}|_{H^{s}(T)}
$$

for all $\zeta \in\left[H^{s}(T)\right]^{2}$, where $h_{T}=\operatorname{diam} T$, and the positive constant $C_{T}$ depends on the minimum angle of $T$ (and also on $s$ when $s$ is close to $1 / 2$ ). Here and below we use $C$ (with or without subscripts) to denote a generic positive constant that can take different values at different occurrences.

Since the solution $\stackrel{\circ}{u}$ of the RTHM Maxwell equations belongs to $\left[H^{s}(\Omega)\right]^{2}$ for some $s>\frac{1}{2}$ (cf. [15] and Remark 4.1 below), we can define a global interpolant of $\stackrel{\circ}{u}$ by

$$
\left(\Pi_{h} \stackrel{\circ}{\boldsymbol{u}}\right)_{T}=\Pi_{T} \stackrel{\circ}{\boldsymbol{u}_{T}} \quad \forall T \in \mathcal{T}_{h},
$$

where $\stackrel{\circ}{\boldsymbol{u}}_{T}=\left.\stackrel{\circ}{\boldsymbol{u}}\right|_{T}$. It follows from (2.3) and $\nabla \cdot \stackrel{\circ}{\boldsymbol{u}}=0$ that $\Pi_{h} \stackrel{\circ}{\boldsymbol{u}} \in V_{h}$.

In order to recover optimal order convergence for the finite element method introduced in Section 3, the triangulation $\mathcal{T}_{h}$ is graded around the corners $c_{1}, \ldots, c_{L}$ of $\Omega$ so that

$$
h_{T} \approx h \Phi_{\mu}(T) \quad \forall T \in \mathcal{T}_{h},
$$

where $h$ is the mesh parameter, $\mu=\left(\mu_{1}, \ldots, \mu_{L}\right)$, and 


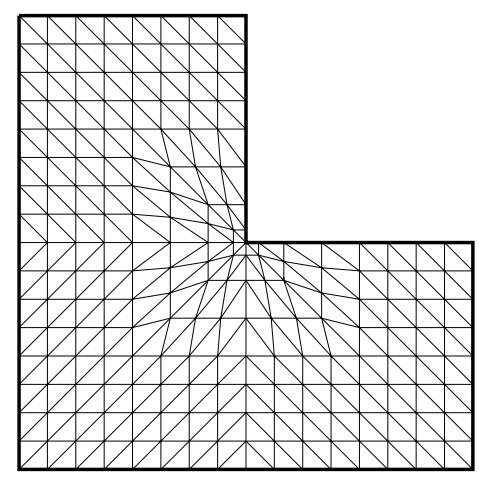

Figure 2.1. A graded mesh on an $L$-shaped domain

$$
\Phi_{\mu}(T)=\Pi_{\ell=1}^{L}\left|c_{\ell}-c_{T}\right|^{1-\mu_{\ell}} .
$$

The point $c_{T}$ is the center of $T$ and $\mu_{\ell}(1 \leq \ell \leq L)$ is the grading parameter at the corner $c_{\ell}$, whose choice will be addressed in Section 4 . Observe that

$$
\Phi_{\mu}(T) \lesssim 1 \quad \forall T \in \mathcal{T}_{h}
$$

To prevent the proliferation of constants, henceforth we also use the notation $A \lesssim B$ (or $B \gtrsim A$ ) to represent the inequality $A \leq$ (constant) $\times B$, where the positive constant is independent of the mesh parameter $h$ and the parameter $\epsilon$ that appears in some of the elliptic regularity estimates. The statement $A \approx B$ is equivalent to $A \lesssim B$ and $B \lesssim A$.

The construction of $\mathcal{T}_{h}$ that satisfies condition (2.5) is discussed for example in [1, 4. (A graded mesh on an $L$-shaped domain is depicted in Figure 2.1 where the grading parameter equals $1 / 3$ at the re-entrant corner and 1 at all the other corners.) Here we note that for a fixed $\mu=\left(\mu_{1}, \ldots, \mu_{L}\right)$, the family $\mathcal{T}_{h}$ is regular (i.e., it satisfies the minimum angle condition) and that (2.5)-(2.7) in particular imply

$$
\begin{array}{ll}
h_{T} \lesssim h & \forall T \in \mathcal{T}_{h}, \\
h_{T} \approx h^{1 / \mu_{\ell}} & \text { if } \quad c_{\ell} \in \partial T .
\end{array}
$$

\section{Discretization And An ABStract ERror estimate}

Before we discretize (1.3), we first introduce some notation. The piecewise curl of $\boldsymbol{v} \in V_{h}$ is given by

$$
\left.\left(\nabla_{h} \times \boldsymbol{v}\right)\right|_{T}=\nabla \times \boldsymbol{v}_{T} \quad \forall \boldsymbol{v} \in V_{h}, T \in \mathcal{T}_{h}
$$

the set of the interior (resp. boundary) edges of $\mathcal{T}_{h}$ is denoted by $\mathcal{E}_{h}^{i}$ (resp. $\mathcal{E}_{h}^{b}$ ), and $\mathcal{E}_{h}=\mathcal{E}_{h}^{i} \cup \mathcal{E}_{h}^{b}$. We use $m_{e}$ and $|e|$ to denote the midpoint and the length of an edge $e$ respectively.

Let $e \in \mathcal{E}_{h}^{i}$ be shared by the two triangles $T_{1}, T_{2} \in \mathcal{T}_{h}$ (cf. Figure 3.1) and let $\boldsymbol{n}_{1}$ (resp. $\boldsymbol{n}_{2}$ ) be the unit normal of $e$ pointing towards the outside of $T_{1}$ (resp. $T_{2}$ ). We define, on $e$,

$$
\begin{aligned}
\llbracket \boldsymbol{n} \times \boldsymbol{v} \rrbracket & =\boldsymbol{n}_{1} \times\left.\boldsymbol{v}_{T_{1}}\right|_{e}+\boldsymbol{n}_{2} \times\left.\boldsymbol{v}_{T_{2}}\right|_{e}, \\
\llbracket \boldsymbol{n} \cdot \boldsymbol{v} \rrbracket & =\left.\boldsymbol{n}_{1} \cdot \boldsymbol{v}_{T_{1}}\right|_{e}+\left.\boldsymbol{n}_{2} \cdot \boldsymbol{v}_{T_{2}}\right|_{e} .
\end{aligned}
$$




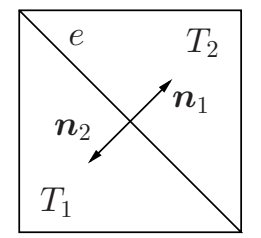

Figure 3.1. Triangles and normals in the definitions of $\llbracket \boldsymbol{n} \times \boldsymbol{v} \rrbracket$ and $\llbracket \boldsymbol{n} \cdot \boldsymbol{v} \rrbracket$

For an edge $e \in \mathcal{E}_{h}^{b}$, we take $\boldsymbol{n}_{e}$ to be the unit normal of $e$ pointing towards the outside of $\Omega$ and define

$$
\llbracket \boldsymbol{n} \times \boldsymbol{v} \rrbracket=\boldsymbol{n}_{e} \times\left.\boldsymbol{v}\right|_{e} .
$$

The discrete problem for the RTHM equations is:

Find $\stackrel{\circ}{\boldsymbol{u}}_{h} \in V_{h}$ such that

$$
a_{h}\left(\stackrel{\circ}{h}_{h}, \boldsymbol{v}\right)=(\boldsymbol{f}, \boldsymbol{v}) \quad \forall \boldsymbol{v} \in V_{h},
$$

where

$$
\begin{array}{r}
a_{h}(\boldsymbol{w}, \boldsymbol{v})=\left(\nabla_{h} \times \boldsymbol{w}, \nabla_{h} \times \boldsymbol{v}\right)-k^{2}(\boldsymbol{w}, \boldsymbol{v}) \\
+\sum_{e \in \mathcal{E}_{h}} \frac{\left[\Phi_{\mu}(e)\right]^{2}}{|e|} \int_{e} \llbracket \boldsymbol{n} \times \boldsymbol{w} \rrbracket \llbracket \boldsymbol{n} \times \boldsymbol{v} \rrbracket d s \\
\quad+\sum_{e \in \mathcal{E}_{h}^{i}} \frac{\left[\Phi_{\mu}(e)\right]^{2}}{|e|} \int_{e} \llbracket \boldsymbol{n} \cdot \boldsymbol{w} \rrbracket \llbracket \boldsymbol{n} \cdot \boldsymbol{v} \rrbracket d s, \\
\Phi_{\mu}(e)=\Pi_{\ell=1}^{L}\left|c_{\ell}-m_{e}\right|^{1-\mu_{\ell}} .
\end{array}
$$

Observe that, by comparing (2.6) and (3.6), we have

$$
\Phi_{\mu}(e) \approx \Phi_{\mu}(T) \quad \text { if } \quad e \subset \partial T .
$$

Remark 3.1. The term involving $\llbracket \boldsymbol{n} \times \boldsymbol{w} \rrbracket \llbracket \boldsymbol{n} \times \boldsymbol{v} \rrbracket$ also appears in discontinuous Galerkin methods for the time-harmonic Maxwell equations [16, 14]. However, its role here is to ensure the consistency of the scheme (3.4) and there is no need for a penalty parameter.

We will measure the discretization error in terms of the norm $\|\cdot\|_{h}$ defined by

$$
\begin{gathered}
\|\boldsymbol{v}\|_{h}^{2}=\left\|\nabla_{h} \times \boldsymbol{v}\right\|_{L_{2}(\Omega)}^{2}+\|\boldsymbol{v}\|_{L_{2}(\Omega)}^{2}+\sum_{e \in \mathcal{E}_{h}} \frac{\left[\Phi_{\mu}(e)\right]^{2}}{|e|}\|\llbracket \boldsymbol{n} \times \boldsymbol{v} \rrbracket\|_{L_{2}(e)}^{2} \\
+\sum_{e \in \mathcal{E}_{h}^{i}} \frac{\left[\Phi_{\mu}(e)\right]^{2}}{|e|}\|\llbracket \boldsymbol{n} \cdot \boldsymbol{v} \rrbracket\|_{L_{2}(e)}^{2} .
\end{gathered}
$$

Observe that

$$
\|\boldsymbol{v}\|_{L_{2}(\Omega)} \leq\|\boldsymbol{v}\|_{h} \quad \forall \boldsymbol{v} \in V_{h}
$$

and $a_{h}(\cdot, \cdot)$ is bounded with respect to $\|\cdot\|_{h}$, i.e.,

$$
a_{h}(\boldsymbol{v}, \boldsymbol{w}) \leq\left(k^{2}+1\right)\|\boldsymbol{v}\|_{h}\|\boldsymbol{w}\|_{h}
$$


for all $\boldsymbol{v}, \boldsymbol{w} \in\left[H_{0}(\operatorname{curl} ; \Omega) \cap H\left(\operatorname{div}^{0} ; \Omega\right)\right]+V_{h}$. Furthermore, we have a trivial Gårding (in)equality,

$$
a_{h}(\boldsymbol{v}, \boldsymbol{v})+\left(k^{2}+1\right)(\boldsymbol{v}, \boldsymbol{v})=\|\boldsymbol{v}\|_{h}^{2}
$$

for all $v \in\left[H_{0}(\operatorname{curl} ; \Omega) \cap H\left(\operatorname{div}^{0} ; \Omega\right)\right]+V_{h}$.

The following lemma provides an abstract discretization error estimate under the assumption that (3.4) is solvable.

Lemma 3.2. Let $\stackrel{\circ}{\boldsymbol{u}} \in H_{0}(\operatorname{curl} ; \Omega) \cap H\left(\operatorname{div}^{0} ; \Omega\right)$ satisfy (1.3) and let $\stackrel{\circ}{u}_{h}$ be a solution of (3.4). It holds that

$$
\begin{aligned}
&\left\|\stackrel{\circ}{\boldsymbol{u}}-\stackrel{\circ}{\boldsymbol{u}}_{h}\right\|_{h} \leq\left(2 k^{2}+3\right) \inf _{\boldsymbol{v} \in V_{h}}\|\stackrel{\circ}{\boldsymbol{u}}-\boldsymbol{v}\|_{h} \\
&+\sup _{\boldsymbol{w} \in V_{h} \backslash\{\boldsymbol{0}\}} \frac{a_{h}\left(\stackrel{\circ}{\boldsymbol{u}}-\stackrel{\circ}{\boldsymbol{u}}_{h}, \boldsymbol{w}\right)}{\|\boldsymbol{w}\|_{h}} \\
& \quad+\left(k^{2}+1\right)\left\|\stackrel{\circ}{\boldsymbol{u}}-\stackrel{\circ}{\boldsymbol{u}}_{h}\right\|_{L_{2}(\Omega)} .
\end{aligned}
$$

Proof. From (3.9) and (3.11) we have

$$
\|\boldsymbol{v}\|_{h}=\frac{a_{h}(\boldsymbol{v}, \boldsymbol{v})}{\|\boldsymbol{v}\|_{h}}+\left(k^{2}+1\right) \frac{(\boldsymbol{v}, \boldsymbol{v})}{\|\boldsymbol{v}\|_{h}} \leq \sup _{\boldsymbol{w} \in V_{h} \backslash\{\mathbf{0}\}} \frac{a_{h}(\boldsymbol{v}, \boldsymbol{w})}{\|\boldsymbol{w}\|_{h}}+\left(k^{2}+1\right)\|\boldsymbol{v}\|_{L_{2}(\Omega)}
$$

for all $\boldsymbol{v} \in V_{h} \backslash\{\mathbf{0}\}$. It follows that

$$
\|\boldsymbol{v}\|_{h} \leq \sup _{\boldsymbol{w} \in V_{h} \backslash\{\mathbf{0}\}} \frac{a_{h}(\boldsymbol{v}, \boldsymbol{w})}{\|\boldsymbol{w}\|_{h}}+\left(k^{2}+1\right)\|\boldsymbol{v}\|_{L_{2}(\Omega)} \quad \forall \boldsymbol{v} \in V_{h} .
$$

Let $\boldsymbol{v} \in V_{h}$ be arbitrary. Using (3.9), (3.10) and (3.13) we find

$$
\begin{aligned}
& \left\|\stackrel{\circ}{\boldsymbol{u}}-\stackrel{\circ}{\boldsymbol{u}}_{h}\right\|_{h} \leq\|\stackrel{\circ}{\boldsymbol{u}}-\boldsymbol{v}\|_{h}+\left\|\boldsymbol{v}-\stackrel{\circ}{\boldsymbol{u}}_{h}\right\|_{h} \\
& \leq\|\stackrel{\circ}{\boldsymbol{u}}-\boldsymbol{v}\|_{h}+\sup _{\boldsymbol{w} \in V_{h} \backslash\{\mathbf{0}\}} \frac{a_{h}\left(\boldsymbol{v}-\stackrel{\circ}{\boldsymbol{u}}_{h}, \boldsymbol{w}\right)}{\|\boldsymbol{w}\|_{h}} \\
& +\left(k^{2}+1\right)\left\|\boldsymbol{v}-\stackrel{\circ}{u}_{h}\right\|_{L_{2}(\Omega)} \\
& \leq\left(2 k^{2}+3\right)\|\stackrel{\circ}{\boldsymbol{u}}-\boldsymbol{v}\|_{h}+\sup _{\boldsymbol{w} \in V_{h} \backslash\{\mathbf{0}\}} \frac{a_{h}\left(\stackrel{\circ}{\boldsymbol{u}}-\stackrel{\circ}{\boldsymbol{u}}_{h}, \boldsymbol{w}\right)}{\|\boldsymbol{w}\|_{h}} \\
& +\left(k^{2}+1\right)\left\|\stackrel{\circ}{\boldsymbol{u}}-\stackrel{\circ}{\boldsymbol{u}}_{h}\right\|_{L_{2}(\Omega)},
\end{aligned}
$$

which implies (3.12).

In what follows we consider $k$ to be fixed and simply write (3.12) as

$$
\left\|\stackrel{\circ}{\boldsymbol{u}}-\stackrel{\circ}{\boldsymbol{u}}_{h}\right\|_{h} \lesssim \inf _{\boldsymbol{v} \in V_{h}}\|\stackrel{\circ}{\boldsymbol{u}}-\boldsymbol{v}\|_{h}+\sup _{\boldsymbol{w} \in V_{h} \backslash\{\mathbf{0}\}} \frac{a_{h}\left(\stackrel{\circ}{\boldsymbol{u}}-\stackrel{\circ}{\boldsymbol{u}}_{h}, \boldsymbol{w}\right)}{\|\boldsymbol{w}\|_{h}}+\left\|\stackrel{\circ}{\boldsymbol{u}}-\stackrel{\circ}{\boldsymbol{u}}_{h}\right\|_{L_{2}(\Omega)} .
$$

Remark 3.3. The first term on the right-hand side of 3.12 measures the approximation property of $V_{h}$ with respect to the norm $\|\cdot\|_{h}$. The second term measures the consistency error of the nonconforming discretization. The third term addresses the indefiniteness of the RTHM equations. 


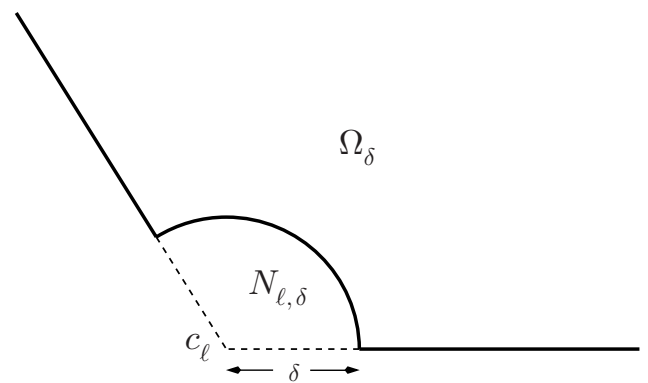

FiguRE 4.1. $\Omega_{\delta}$ and $N_{\ell, \delta}$

\section{Singularities of the RTHM equations and grading Parameters}

Since $Q \boldsymbol{f} \in\left[L_{2}(\Omega)\right]^{2}$ and $\nabla \cdot \stackrel{\circ}{\boldsymbol{u}}=0$, elliptic regularity and (1.5) imply that

$$
\stackrel{\circ}{\boldsymbol{u}} \in\left[H^{2}\left(\Omega_{\delta}\right)\right]^{2},
$$

where $\Omega_{\delta}$ is the domain obtained from $\Omega$ by excising $\delta$-neighborhoods (cf. Figure 4.1) at the corners of $\Omega$, and

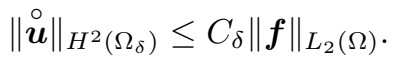

The regularity of $\stackrel{\circ}{\boldsymbol{u}}$ stated in (4.1) allows optimal approximation by the piecewise $P_{1}$ vector fields in $V_{h}$ away from the corners of $\Omega$. Therefore the choices of the grading parameters $\mu_{\ell}$ for $1 \leq \ell \leq L$ are determined by the nature of the singularities of $\stackrel{\circ}{\boldsymbol{u}}$ at the corners of $\Omega$. Details of the discussion below can be found in 2,7 .

Let $(r, \theta)$ be the polar coordinates at $c_{\ell}$ such that the two edges of $\Omega$ emanating from $c_{\ell}$ are given by $\theta=0$ and $\theta=\omega_{\ell}$, where $\omega_{\ell}$ is the interior angle of $\Omega$ at $c_{\ell}$, and

$$
\psi_{\ell, j}(r, \theta)=r^{j\left(\pi / \omega_{\ell}\right)-1}\left[\begin{array}{l}
\sin \left(j\left(\pi / \omega_{\ell}\right)-1\right) \theta \\
\cos \left(j\left(\pi / \omega_{\ell}\right)-1\right) \theta
\end{array}\right] .
$$

In the $\delta$-neighborhood (cf. Figure 4.1)

$$
N_{\ell, \delta}=\left\{x \in \Omega:\left|x-c_{\ell}\right|<\delta\right\}
$$

of $c_{\ell}$ in $\Omega$, we have a singular vector field representation for $\stackrel{\circ}{\boldsymbol{u}}$ :

$$
\stackrel{\circ}{\boldsymbol{u}}=\stackrel{\circ}{\boldsymbol{u}}_{R}+\sum_{j=1}^{J_{\ell}} \kappa_{\ell, j} \boldsymbol{\psi}_{\ell, j} .
$$

The precise form of the representation (4.5) can be divided into several cases. In the first case we have

$$
J_{\ell}=0 \quad \text { and } \quad \stackrel{\circ}{\boldsymbol{u}}_{R}=\stackrel{\circ}{\boldsymbol{u}} \in\left[H^{2-\epsilon}\left(N_{\ell, \delta}\right)\right]^{2} \quad \text { if } \quad \omega_{\ell} \leq \frac{\pi}{2},
$$

where $\epsilon$ is any positive number, and

$$
\|\stackrel{\circ}{u}\|_{H^{2-\epsilon}\left(N_{\ell, \delta}\right)} \leq C_{\ell, \epsilon}\|\boldsymbol{f}\|_{L_{2}(\Omega)} .
$$

In the second case we have

$$
J_{\ell}=1 \quad \text { and } \quad \stackrel{\circ}{\boldsymbol{u}}_{R} \in\left[H^{2}\left(N_{\ell, \delta}\right)\right]^{2} \quad \text { if } \quad \frac{\pi}{2}<\omega_{\ell}<\pi .
$$


Furthermore, it holds that

$$
\left|\kappa_{\ell, 1}\right|+\left\|\stackrel{\circ}{R}_{R}\right\|_{H^{2}\left(N_{\ell, \delta}\right)} \leq C_{\ell}\|\boldsymbol{f}\|_{L_{2}(\Omega)} .
$$

In the third case we have

$$
J_{\ell}=2 \quad \text { and } \quad \stackrel{\circ}{\boldsymbol{u}}_{R} \in\left[H^{2-\epsilon}\left(N_{\ell, \delta}\right)\right]^{2} \quad \text { if } \quad \pi<\omega_{\ell} \leq \frac{3}{2} \pi,
$$

where $\epsilon$ is any positive number, and

$$
\left|\kappa_{\ell, 1}\right|+\left|\kappa_{\ell, 2}\right|+\left\|\stackrel{\circ}{R}_{R}\right\|_{H^{2-\epsilon}\left(N_{\ell, \delta}\right)} \leq C_{\ell, \epsilon}\|\boldsymbol{f}\|_{L_{2}(\Omega)} .
$$

In the final case we have

$$
J_{\ell}=3 \quad \text { and } \quad \stackrel{\circ}{u}_{R} \in\left[H^{2}\left(N_{\ell, \delta}\right)\right]^{2} \quad \text { if } \quad \frac{3}{2} \pi<\omega_{\ell}<2 \pi,
$$

and

$$
\left|\kappa_{\ell, 1}\right|+\left|\kappa_{\ell, 2}\right|+\left|\kappa_{\ell, 3}\right|+\left\|\stackrel{\circ}{\circ}_{R}\right\|_{H^{2}\left(N_{\ell, \delta}\right)} \leq C_{\ell}\|\boldsymbol{f}\|_{L_{2}(\Omega)} .
$$

In the first case where $\omega_{\ell} \leq \frac{\pi}{2}$, there is no need for a graded mesh around $c_{\ell}$ and we can take $\mu_{\ell}=1$. For the other three cases we take $\mu_{\ell}$ to be less than $\frac{\pi}{2 \omega_{\ell}}$, i.e., the grading parameters $\mu_{\ell}$ for $1 \leq \ell \leq L$ satisfy

$$
\begin{array}{llll}
\mu_{\ell}=1 & \text { if } & \omega_{\ell} \leq \frac{\pi}{2}, \\
\mu_{\ell}<\frac{\pi}{2 \omega_{\ell}} & \text { if } & \omega_{\ell}>\frac{\pi}{2} .
\end{array}
$$

The key observation is that, for $\omega_{\ell}>\frac{\pi}{2}$, (4.3) and (4.14) imply

$$
\sum_{T \in \mathcal{T}_{h}, T \subset N_{\ell, \delta}} \int_{T}\left|c_{\ell}-x\right|^{4\left(1-\mu_{\ell}\right)}\left|D^{2} \boldsymbol{\psi}_{\ell, j}\right|^{2} d x<\infty,
$$

where $\left|D^{2} \boldsymbol{v}\right|^{2}=\sum_{i, j, k=1}^{2}\left(\frac{\partial^{2} v_{i}}{\partial x_{j} \partial x_{k}}\right)^{2}$, because

$$
\int_{0}^{1} r^{4\left(1-\mu_{\ell}\right)} r^{2\left(\left(\pi / \omega_{\ell}\right)-3\right)} r d r<\infty \quad \text { if } \quad \mu_{\ell}<\frac{\pi}{2 \omega_{\ell}}
$$

Remark 4.1. The singular vector field representation (4.5) implies $\stackrel{\circ}{\boldsymbol{u}} \in\left[H^{s}\left(N_{\ell, \delta}\right)\right]^{2}$ for any $s<\frac{\pi}{\omega_{\ell}}$. Therefore $\stackrel{\circ}{\boldsymbol{u}}$ belongs to $\left[H^{s}(\Omega)\right]^{2}$ for any $s<\min _{1 \leq \ell \leq L} \frac{\pi}{\omega_{\ell}}$. In particular, we can choose $s$ to be strictly greater than $\frac{1}{2}$.

Remark 4.2. In the case where $\omega_{\ell}>\frac{\pi}{2}$, since $2 \mu_{\ell}<\frac{\pi}{\omega_{\ell}}$, it follows from well-known results [13, 11] for the singular vector fields $\boldsymbol{\psi}_{\ell, j}$ that $\stackrel{\circ}{\boldsymbol{u}} \in\left[H^{2 \mu_{\ell}}\left(N_{\ell, \delta}\right)\right]^{2}$ and, in view of (4.5) and (4.8)-(4.13), the following regularity estimate is valid:

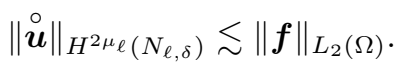

Remark 4.3. When $\omega$ is larger than $\pi / 2$, the solution $\stackrel{\circ}{\boldsymbol{u}}$ does not belong to $H^{2}\left(N_{\ell, \delta}\right)$ since $\pi / \omega_{\ell}<2$ (cf. Remark 4.1). This is the reason why local refinement is required when $\omega_{\ell}>\pi / 2$. Otherwise the results in Lemma 5.2 and Lemma 6.5 will fail to hold, and the optimal error estimates for the energy norm and the $L_{2}$ norm in Theorem 6.6 are no longer valid. 


\section{Preliminary estimates}

In this section we establish some preliminary estimates that are needed for the convergence analysis in Section [6] We assume that a positive $\delta$ has been chosen. Let $\mathcal{T}_{h, \ell}$ be the set of the triangles of $\mathcal{T}_{h}$ that share the corner $c_{\ell}$ as a common vertex. Without loss of generality, we may assume that $h \ll \delta$ and hence $T \in$ $\mathcal{T}_{h, \ell} \Rightarrow T \subset N_{\ell, \delta}$, where $N_{\ell, \delta}$ is the neighborhood of $c_{\ell}$ defined in (4.4). We will use the notation $\mathcal{T}_{h}^{c}=\bigcup_{\ell=1}^{L} \mathcal{T}_{h, \ell}$ and $\mathcal{T}_{h}^{i}=\mathcal{T}_{h} \backslash \mathcal{T}_{h}^{c}$ in the proofs of the first two lemmas.

Lemma 5.1. Let $\stackrel{\circ}{\boldsymbol{u}} \in H_{0}(\operatorname{curl} ; \Omega) \cap H\left(\operatorname{div}^{0} ; \Omega\right)$ be the solution of (1.3). It holds that

$$
\left\|\stackrel{\circ}{\boldsymbol{u}}-\Pi_{h} \stackrel{\circ}{\boldsymbol{u}}\right\|_{L_{2}(\Omega)} \leq C_{\epsilon} h^{2-\epsilon}\|\boldsymbol{f}\|_{L_{2}(\Omega)} \quad \text { for any } \quad \epsilon>0 .
$$

Proof. We can write

$$
\left\|\stackrel{\circ}{\|}-\Pi_{h} \stackrel{\circ}{\boldsymbol{u}}\right\|_{L_{2}(\Omega)}^{2}=\sum_{T \in \mathcal{T}_{h}^{i}}\left\|\stackrel{\circ}{\boldsymbol{u}}-\Pi_{T} \stackrel{\circ}{\boldsymbol{u}}\right\|_{L_{2}(T)}^{2}+\sum_{T \in \mathcal{T}_{h}^{c}}\left\|\stackrel{\circ}{\boldsymbol{u}}-\Pi_{T} \stackrel{\circ}{\boldsymbol{u}}\right\|_{L_{2}(T)}^{2}
$$

We have, by (2.4) (with $s=2$ ), (2.8), (4.1) and (4.2),

$$
\sum_{T \in \mathcal{T}_{h}^{i}, T \not \subset \bigcup_{\ell=1}^{L} N_{\ell, \delta}}\left\|\stackrel{\circ}{\boldsymbol{u}}-\Pi_{T} \stackrel{\circ}{u}\right\|_{L_{2}(T)}^{2} \lesssim h^{4}\|\boldsymbol{f}\|_{L_{2}(\Omega)}^{2} .
$$

On the other hand, near a corner $c_{\ell}$ of $\Omega$ we can use (4.5) to obtain

$$
\begin{aligned}
& \sum_{T \in \mathcal{T}_{h}^{i}, T \subset N_{\ell, \delta}}\left\|\stackrel{\circ}{\boldsymbol{u}}-\Pi_{T} \stackrel{\circ}{\boldsymbol{u}}\right\|_{L_{2}(T)}^{2} \\
& \lesssim \sum_{T \in \mathcal{T}_{h}^{i}, T \subset N_{\ell, \delta}}\left[\left\|\stackrel{\circ}{\boldsymbol{u}}_{R}-\Pi_{T}{\stackrel{\circ}{\boldsymbol{u}_{R}}}_{R}\right\|_{L_{2}(T)}^{2}\right. \\
& \left.\quad+\sum_{j=1}^{J_{\ell}}\left|\kappa_{\ell, j}\right|^{2}\left\|\boldsymbol{\psi}_{\ell, j}-\Pi_{T} \boldsymbol{\psi}_{\ell, j}\right\|_{L_{2}(T)}^{2}\right] .
\end{aligned}
$$

The estimates (2.4) (with $s=2-\epsilon$ ), (2.8), (4.7), (4.9), (4.11) and (4.13) imply

$$
\sum_{T \in \mathcal{T}_{h}^{i}, T \subset N_{\ell, \delta}}\left\|{\stackrel{\circ}{\boldsymbol{u}_{R}}}_{R}-\Pi_{T}{\stackrel{\circ}{\boldsymbol{u}_{R}}}_{R}^{2}\right\|_{L_{2}(T)} \leq C_{\epsilon} h^{4-\epsilon}\|\boldsymbol{f}\|_{L_{2}(\Omega)}^{2}
$$

for any $\epsilon>0$.

Note that (2.5) and the regularity of $\mathcal{T}_{h}$ imply that

$$
\left|c_{\ell}-c_{T}\right| \approx\left|c_{\ell}-x\right| \quad \forall x \in T \in \mathcal{T}_{h}^{i} \quad \text { and } \quad T \subset N_{\ell, \delta},
$$

and hence

$$
\Phi_{\mu}(T) \approx\left|c_{\ell}-x\right|^{1-\mu_{\ell}} \quad \forall x \in T \in \mathcal{T}_{h}^{i} \quad \text { and } \quad T \subset N_{\ell, \delta}
$$


Using (2.4) (with $s=2$ ), (2.5), (4.15) and (5.6) we obtain the following estimate for the term involving the singular vector fields:

$$
\begin{gathered}
\sum_{T \in \mathcal{T}_{h}^{i}, T \subset N_{\ell, \delta}}\left\|\boldsymbol{\psi}_{\ell, j}-\Pi_{T} \boldsymbol{\psi}_{\ell, j}\right\|_{L_{2}(T)}^{2} \lesssim \sum_{T \in \mathcal{T}_{h}^{i}, T \subset N_{\ell, \delta}} h_{T}^{4}\left|\boldsymbol{\psi}_{\ell, j}\right|_{H^{2}(T)}^{2} \\
\approx h^{4} \sum_{T \in \mathcal{T}_{h}^{i}, T \subset N_{\ell, \delta}}\left[\Phi_{\mu}(T)\right]^{4}\left|\boldsymbol{\psi}_{\ell, j}\right|_{H^{2}(T)}^{2} \\
\approx h^{4} \sum_{T \in \mathcal{T}_{h}^{i}, T \subset N_{\ell, \delta}} \int_{T}\left|c_{\ell}-x\right|^{4\left(1-\mu_{\ell}\right)}\left|D^{2} \boldsymbol{\psi}_{\ell, j}\right|^{2} d x \lesssim h^{4} .
\end{gathered}
$$

Combining (4.9), (4.11), (4.13), (5.3)-(5.5) and (5.7), we arrive at

$$
\sum_{T \in \mathcal{T}_{h}^{i}}\left\|\stackrel{\circ}{\boldsymbol{u}}-\Pi_{T} \stackrel{\circ}{\boldsymbol{u}}\right\|_{L_{2}(T)}^{2} \leq C_{\epsilon} h^{4-\epsilon}\|\boldsymbol{f}\|_{L_{2}(\Omega)}^{2} \quad \text { for any } \epsilon>0 .
$$

It remains to estimate the second term on the right-hand side of (5.2). For the case where $\omega_{\ell} \leq \frac{\pi}{2}$, it follows from (2.4) (with $s=2-\epsilon$ ) and (4.5)-(4.7) that

$$
\sum_{T \in \mathcal{T}_{h, \ell}}\left\|\stackrel{\circ}{\boldsymbol{u}}-\Pi_{T} \stackrel{\circ}{\boldsymbol{u}}\right\|_{L_{2}(T)}^{2} \leq C_{\epsilon} h^{4-\epsilon}\|\boldsymbol{f}\|_{L_{2}(\Omega)}^{2} \quad \text { if } \quad \omega_{\ell} \leq \frac{\pi}{2} .
$$

For the case where $\omega_{\ell}>\frac{\pi}{2}$, since $\stackrel{\circ}{\boldsymbol{u}} \in\left[H^{2 \mu_{\ell}}(\Omega)\right]^{2}$ (cf. Remark 4.2), we obtain from (2.4) (with $\left.s=2 \mu_{\ell}\right)$, (2.9) and (4.16) the estimate

$$
\sum_{T \in \mathcal{T}_{h, \ell}}\left\|\stackrel{\circ}{\boldsymbol{u}}-\Pi_{T} \stackrel{\circ}{\boldsymbol{u}}\right\|_{L_{2}(T)}^{2} \lesssim h_{T}^{4 \mu_{\ell}}\|\boldsymbol{f}\|_{L_{2}(\Omega)}^{2} \approx h^{4}\|\boldsymbol{f}\|_{L_{2}(\Omega)}^{2}
$$

if $\omega_{\ell}>\pi / 2$. Combining (5.9) and (5.10), we have

$$
\sum_{T \in \mathcal{T}_{h}^{c}}\left\|\stackrel{\circ}{\boldsymbol{u}}-\Pi_{T} \stackrel{\circ}{\boldsymbol{u}}\right\|_{L_{2}(\Omega)}^{2} \leq C_{\epsilon} h^{4-\epsilon}\|\boldsymbol{f}\|_{L_{2}(\Omega)}^{2} \quad \text { for any } \epsilon>0 .
$$

The estimate (5.1) follows from (5.2), (5.8) and (5.11).

Lemma 5.2. Let $\stackrel{\circ}{\boldsymbol{u}} \in H_{0}(\operatorname{curl} ; \Omega) \cap H\left(\operatorname{div}^{0} ; \Omega\right)$ be the solution of (1.3). It holds that

$$
\sum_{e \in \mathcal{E}_{h}} \frac{\left[\Phi_{\mu}(e)\right]^{2}}{|e|}\left\|\llbracket \stackrel{\circ}{\boldsymbol{u}}-\Pi_{h} \stackrel{\circ}{\boldsymbol{u}} \rrbracket\right\|_{L_{2}(e)}^{2} \leq C_{\epsilon} h^{2-\epsilon}\|\boldsymbol{f}\|_{L_{2}(\Omega)}^{2}
$$

for any $\epsilon>0$, where $\llbracket \stackrel{\circ}{\boldsymbol{u}}-\Pi_{h} \stackrel{\circ}{\boldsymbol{u}} \rrbracket$ is the jump of $\stackrel{\circ}{\boldsymbol{u}}-\Pi_{h} \boldsymbol{u}$ across the interior edges of $\mathcal{T}_{h}$ and $\llbracket \stackrel{\circ}{\boldsymbol{u}}-\Pi_{h} \stackrel{\circ}{\mathbf{u}} \rrbracket=\stackrel{\circ}{\boldsymbol{u}}-\Pi_{h} \stackrel{\circ}{\boldsymbol{u}}$ on the boundary edges of $\mathcal{T}_{h}$.

Proof. Let $e \in \mathcal{E}_{h}$ and $\mathcal{T}_{e}$ be the set of the triangles in $\mathcal{T}_{h}$ having $e$ as an edge. We have

$$
\frac{1}{|e|}\left\|\llbracket \stackrel{\circ}{\boldsymbol{u}}-\Pi_{h} \stackrel{\circ}{\boldsymbol{u}} \rrbracket\right\|_{L_{2}(e)}^{2} \lesssim \sum_{T \in \mathcal{T}_{e}}|e|^{-1}\left\|\stackrel{\circ}{\boldsymbol{u}}-\Pi_{T} \stackrel{\circ}{\boldsymbol{u}}\right\|_{L_{2}(e)}^{2} .
$$

If $T \in \mathcal{T}_{e}$ belongs to $\mathcal{T}_{h}^{i}$, we can use the trace theorem (with scaling) and the Bramble-Hilbert lemma [3, 5] to obtain

$$
\begin{aligned}
|e|^{-1}\left\|\stackrel{\circ}{\boldsymbol{u}}-\Pi_{T} \stackrel{\circ}{\boldsymbol{u}}\right\|_{L_{2}(e)}^{2} & \lesssim h_{T}^{-2}\left\|\stackrel{\circ}{\boldsymbol{u}}-\Pi_{T} \stackrel{\circ}{\boldsymbol{u}}\right\|_{L_{2}(T)}^{2}+\left|\stackrel{\circ}{\boldsymbol{u}}-\Pi_{T} \stackrel{\circ}{\boldsymbol{u}}\right|_{H^{1}(T)}^{2} \\
& \lesssim\left|\stackrel{\circ}{\boldsymbol{u}}-\Pi_{T} \stackrel{\circ}{\boldsymbol{u}}\right|_{H^{1}(T)}^{2}
\end{aligned}
$$


If $T \in \mathcal{T}_{\ell}$ belongs to $\mathcal{T}_{h, \ell}$ and $\omega_{\ell} \leq \frac{\pi}{2}$, then we have, by (2.4) (with $s=2-\epsilon$ ), (4.5) and (4.6),

$$
\begin{aligned}
|e|^{-1}\left\|\stackrel{\circ}{\boldsymbol{u}}-\Pi_{T} \stackrel{\circ}{u}\right\|_{L_{2}(e)}^{2} & \lesssim h_{T}^{-2}\left\|\stackrel{\circ}{\boldsymbol{u}}-\Pi_{T} \stackrel{\circ}{\boldsymbol{u}}\right\|_{L_{2}(T)}^{2}+\mid \stackrel{\circ}{\boldsymbol{u}}-\Pi_{T} \stackrel{\circ}{\boldsymbol{u}_{H^{1}(T)}^{2}} \\
& \leq C_{\epsilon} h_{T}^{2(1-\epsilon)}\left|\stackrel{\circ}{\boldsymbol{u}^{2}}\right|_{H^{2-\epsilon}(T)}^{2}
\end{aligned}
$$

for any $\epsilon>0$.

On the other hand, if $T \in \mathcal{T}_{\ell}$ belongs to $\mathcal{T}_{h, \ell}$ and $\omega_{\ell}>\frac{\pi}{2}$, we have, by the trace theorem (with scaling) and (2.4) (with $s=2 \mu_{\ell}$ (cf. Remark 4.2)),

$$
\begin{aligned}
|e|^{-1}\left\|\stackrel{\circ}{\boldsymbol{u}}-\Pi_{T} \stackrel{\circ}{\boldsymbol{u}}\right\|_{L_{2}(e)}^{2} \lesssim & h_{T}^{-2}\left\|\stackrel{\circ}{\boldsymbol{u}}-\Pi_{T} \stackrel{\circ}{\boldsymbol{u}}\right\|_{L_{2}(T)}^{2} \\
& \quad+h_{T}^{2\left(\min \left(2 \mu_{\ell}, 1\right)-1\right)}\left|\stackrel{\circ}{\boldsymbol{u}}-\Pi_{T} \stackrel{\circ}{ } \boldsymbol{i}\right|_{H^{\min \left(2 \mu_{\ell}, 1\right)}(T)}^{2} \\
\lesssim & h_{T}^{2\left(2 \mu_{\ell}-1\right)}|\stackrel{\circ}{\boldsymbol{u}}|_{H^{2 \mu_{\ell}(T)}}^{2}
\end{aligned}
$$

It follows from (3.7) and (5.13) $-(5.16)$ that

$$
\begin{aligned}
& \sum_{e \in \mathcal{E}_{h}} \frac{\left[\Phi_{\mu}(e)\right]^{2}}{|e|}\left\|\llbracket \stackrel{\circ}{\boldsymbol{u}}-\Pi_{h} \stackrel{\circ}{\boldsymbol{u}} \rrbracket\right\|_{L_{2}(e)}^{2} \\
& \leq C_{\epsilon}\left\{\sum_{T \in \mathcal{T}_{h}^{i}}\left[\Phi_{\mu}(T)\right]^{2}\left|\stackrel{\circ}{\boldsymbol{u}}-\Pi_{T} \stackrel{\circ}{\boldsymbol{u}}\right|_{H^{1}(T)}^{2}\right. \\
& +\sum_{\omega_{\ell} \leq \frac{\pi}{2}} \sum_{T \in \mathcal{T}_{h, \ell}}\left[\Phi_{\mu}(T)\right]^{2} h_{T}^{2(1-\epsilon)}|\stackrel{\circ}{u}|_{H^{2-\epsilon}(T)}^{2} \\
& \left.+\sum_{\omega_{\ell}>\frac{\pi}{2}} \sum_{T \in \mathcal{T}_{h, \ell}}\left[\Phi_{\mu}(T)\right]^{2} h_{T}^{2\left(2 \mu_{\ell}-1\right)}\left|{ }^{\circ}\right|_{H^{2 \mu_{\ell}(T)}}^{2}\right\}
\end{aligned}
$$

for an $\epsilon>0$. As in the proof of Lemma 5.1 the three terms on the right-hand side of (5.17) can be analyzed as follows.

We can write the first term as

$$
\begin{aligned}
& \sum_{T \in \mathcal{T}_{h}^{i}}\left[\Phi_{\mu}(T)\right]^{2}\left|\stackrel{\circ}{\boldsymbol{u}}-\Pi_{T} \stackrel{\circ}{\boldsymbol{u}}\right|_{H^{1}(T)}^{2} \\
& =\sum_{T \in \mathcal{T}_{h}^{i}, T \not \subset \bigcup_{\ell=1}^{L}}\left[\Phi_{\mu}(T)\right]^{2}\left|\stackrel{\circ}{N_{\ell, \delta}}-\Pi_{T} \stackrel{\circ}{ }\right|_{H^{1}(T)}^{2} \\
& \quad \\
& \quad \sum_{T \in \mathcal{T}_{h}^{i}, T \subset \bigcup_{\ell=1}^{L}}\left[\Phi_{\mu}(T)\right]^{2}\left|\stackrel{\circ}{N_{\ell, \delta}}-\Pi_{T} \stackrel{\circ}{ }\right|_{H^{1}(T)}^{2} .
\end{aligned}
$$

It follows from (2.4) (with $s=2$ ), (2.5), (2.7), (2.8), (4.1) and (4.2) that

$$
\sum_{T \in \mathcal{T}_{h}^{i}, T \not \subset \bigcup_{\ell=1}^{L}, N_{\ell, \delta}}\left[\Phi_{\mu}(T)\right]^{2}\left|\stackrel{\circ}{\mathbf{u}}-\Pi_{T} \stackrel{\circ}{u}\right|_{H^{1}(T)}^{2} \lesssim h^{2}\|\boldsymbol{f}\|_{L_{2}(\Omega)}^{2} .
$$


Near a corner $c_{\ell}$ of $\Omega$, we can use (4.5) to obtain

$$
\begin{aligned}
& \sum_{T \in \mathcal{T}_{h}^{i}, T \subset N_{\ell, \delta}}\left[\Phi_{\mu}(T)\right]^{2}\left|\stackrel{\circ}{\boldsymbol{u}}-\Pi_{T} \stackrel{\circ}{\boldsymbol{u}}\right|_{H^{1}(T)}^{2} \\
& \lesssim \sum_{T \in \mathcal{T}_{h}^{i}, T \subset N_{\ell, \delta}}\left[\Phi_{\mu}(T)\right]^{2}\left[\mid \stackrel{\circ}{R}_{R}-\Pi_{T}{\stackrel{\circ}{\boldsymbol{u}_{R}}}_{H^{1}(T)}^{2}\right. \\
& \left.\quad+\sum_{j=1}^{J_{\ell}}\left|\kappa_{\ell, j}\right|^{2}\left|\boldsymbol{\psi}_{\ell, j}-\Pi_{T} \boldsymbol{\psi}_{\ell, j}\right|_{H^{1}(T)}^{2}\right] .
\end{aligned}
$$

From (2.4) (with $s=2-\epsilon$ ), (2.5), (2.7), (4.7), (4.9), (4.11), (4.13), we have

$$
\sum_{T \in \mathcal{T}_{h}^{i}, T \subset N_{\ell, \delta}}\left[\Phi_{\mu}(T)\right]^{2} \mid{\stackrel{\circ}{\boldsymbol{u}_{R}}}_{R}-\Pi_{T}{\stackrel{\circ}{\boldsymbol{u}_{R}}}_{H_{H^{1}(T)}^{2}} \lesssim C_{\epsilon} h^{2-\epsilon}\|\boldsymbol{f}\|_{L_{2}(\Omega)}^{2} .
$$

Furthermore, (2.4) (with $s=2$ ) and the arguments in the derivation of (5.7) imply that

$$
\begin{aligned}
\sum_{T \in \mathcal{T}_{h}^{i}, T \subset N_{\ell, \delta}}\left[\Phi_{\mu}(T)\right]^{2}\left|\boldsymbol{\psi}_{\ell, j}-\Pi_{T} \boldsymbol{\psi}_{\ell, j}\right|_{H^{1}(T)}^{2} & \\
& \lesssim \sum_{T \in \mathcal{T}_{h}^{i}, T \subset N_{\ell, \delta}}\left[\Phi_{\mu}(T)\right]^{2} h_{T}^{2}\left|\boldsymbol{\psi}_{\ell, j}\right|_{H^{2}(T)}^{2} \\
& \approx h^{2} \sum_{T \in \mathcal{T}_{h}^{i}, T \subset N_{\ell, \delta}}\left[\Phi_{\mu}(T)\right]^{4}\left|\boldsymbol{\psi}_{\ell, j}\right|_{H^{2}(T)}^{2} \lesssim h^{2} .
\end{aligned}
$$

Combining (4.7), (4.9), (4.11), (4.13), and (5.18)-(5.22), we arrive at the estimate

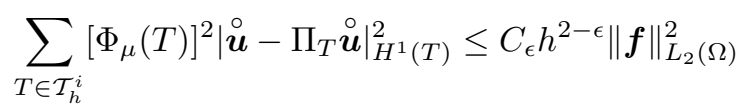

for any $\epsilon>0$.

Next we bound the second term on the right-hand side of (5.17) using the estimates (2.7), (2.8) and (4.5)-(4.7):

$$
\sum_{\omega_{\ell} \leq \frac{\pi}{2}} \sum_{T \in \mathcal{T}_{h, \ell}}\left[\Phi_{\mu}(T)\right]^{2} h_{T}^{2(1-\epsilon)}|\stackrel{\circ}{u}|_{H^{2-\epsilon}(T)}^{2} \leq C_{\epsilon} h^{2(1-\epsilon)}\|\boldsymbol{f}\|_{L_{2}(\Omega)}^{2}
$$

for any $\epsilon>0$.

Finally we derive from (2.5), (2.9) and (4.16) the following estimate for the third term on the right-hand side of (5.17):

$$
\begin{aligned}
\sum_{\omega_{\ell}>\frac{\pi}{2}} & \sum_{T \in \mathcal{T}_{h}, \ell}\left[\Phi_{\mu}(T)\right]^{2} h_{T}^{2\left(2 \mu_{\ell}-1\right)}\left|\stackrel{\circ}{\left.\right|^{2}}\right|_{H^{2 \mu_{\ell}(T)}}^{2} \\
& \approx \sum_{\omega_{\ell}>\frac{\pi}{2}} \sum_{T \in \mathcal{T}_{h, \ell}} h^{-2} h_{T}^{4 \mu_{\ell}}\left|\stackrel{\circ}{\boldsymbol{u}^{2}}\right|_{H^{2 \mu_{\ell}(T)}}^{2} \lesssim h^{2}\|\boldsymbol{f}\|_{L_{2}(\Omega)}^{2} .
\end{aligned}
$$

The estimate (5.12) follows from (5.17) and (5.23)-(5.25).

Lemma 5.3. It holds that

$$
\sum_{e \in \mathcal{E}_{h}}|e|\left[\Phi_{\mu}(e)\right]^{-2}\left\|\eta-\bar{\eta}_{T_{e}}\right\|_{L_{2}(e)}^{2} \lesssim h^{2}|\eta|_{\left.H^{1}(\Omega)\right)}^{2} \quad \forall \eta \in H^{1}(\Omega),
$$


where

$$
\bar{\eta}_{T_{e}}=\frac{1}{\left|T_{e}\right|} \int_{T_{e}} \eta d x
$$

is the mean of $\eta$ over $T_{e}$, one of the triangles in $\mathcal{T}_{h}$ that has e as an edge.

Proof. This is the consequence of (2.5), (3.7), the trace theorem (with scaling) and the Bramble-Hilbert lemma [3]:

$$
\begin{aligned}
\sum_{e \in \mathcal{E}_{h}}|e| & {\left[\Phi_{\mu}(e)\right]^{-2}\left\|\eta-\bar{\eta}_{T_{e}}\right\|_{L_{2}(e)}^{2} } \\
& \lesssim \sum_{e \in \mathcal{E}_{h}}\left[\Phi_{\mu}(T)\right]^{-2}\left(\left\|\eta-\bar{\eta}_{T_{e}}\right\|_{L_{2}\left(T_{e}\right)}^{2}+h_{T}^{2}\left|\eta-\bar{\eta}_{T_{e}}\right|_{H^{1}\left(T_{e}\right)}^{2}\right) \\
& \lesssim \sum_{e \in \mathcal{E}_{h}}\left[\Phi_{\mu}(T)\right]^{-2} h_{T}^{2}|\eta|_{H^{1}\left(T_{e}\right)}^{2} \lesssim h^{2}|\eta|_{H^{1}(\Omega)}^{2} .
\end{aligned}
$$

Recall that $Q$ is the orthogonal projection from $L_{2}(\Omega)$ onto $H\left(\operatorname{div}^{0} ; \Omega\right)$.

Lemma 5.4. The following estimate is valid:

$$
\|\boldsymbol{v}-Q \boldsymbol{v}\|_{L_{2}(\Omega)} \lesssim h\|\boldsymbol{v}\|_{h} \quad \forall \boldsymbol{v} \in\left[H_{0}(\operatorname{curl} ; \Omega) \cap H\left(\operatorname{div}^{0} ; \Omega\right)\right]+V_{h} .
$$

Proof. Let $\boldsymbol{v} \in\left[H_{0}(\operatorname{curl} ; \Omega) \cap H\left(\operatorname{div}^{0} ; \Omega\right)\right]+V_{h}$ be arbitrary. Since $\boldsymbol{v}-Q \boldsymbol{v}$ belongs to $\nabla H_{0}^{1}(\Omega)$, the orthogonal complement of $H\left(\operatorname{div}^{0} ; \Omega\right)$ in $\left[L_{2}(\Omega)\right]^{2}$, we have by duality,

$$
\begin{aligned}
\|\boldsymbol{v}-Q \boldsymbol{v}\|_{L_{2}(\Omega)} & =\sup _{\eta \in H_{0}^{1}(\Omega) \backslash\{0\}} \frac{(\boldsymbol{v}-Q \boldsymbol{v}, \nabla \eta)}{\|\nabla \eta\|_{L_{2}(\Omega)}} \\
& =\sup _{\eta \in H_{0}^{1}(\Omega) \backslash\{0\}} \frac{(\boldsymbol{v}, \nabla \eta)}{\|\nabla \eta\|_{L_{2}(\Omega)}} .
\end{aligned}
$$

Let $\eta \in H_{0}^{1}(\Omega)$ be arbitrary. Since $\nabla \cdot \boldsymbol{v}=0$ on each triangle $T \in \mathcal{T}_{h}$, we find using integration by parts and the fact that on each $e \in \mathcal{E}_{h}^{i}$ the jump $\llbracket \boldsymbol{n} \cdot \boldsymbol{v} \rrbracket$ is a linear polynomial that vanishes at the midpoint,

$$
\begin{aligned}
(\boldsymbol{v}, \nabla \eta) & =\sum_{T \in \mathcal{T}_{h}} \int_{T} \boldsymbol{v} \cdot \nabla \eta d x \\
& =\sum_{e \in \mathcal{E}_{h}^{i}} \int_{e} \eta \llbracket \boldsymbol{n} \cdot \boldsymbol{v} \rrbracket d s \\
& =\sum_{e \in \mathcal{E}_{h}^{i}} \int_{e}\left(\eta-\bar{\eta}_{T_{e}}\right) \llbracket \boldsymbol{n} \cdot \boldsymbol{v} \rrbracket d s,
\end{aligned}
$$

where $\bar{\eta}_{T_{e}}$ is defined in (5.26). It then follows from the Cauchy-Schwarz inequality, (3.8) and Lemma 5.3 that

$$
\begin{gathered}
(\boldsymbol{v}, \nabla \eta) \leq\left[\sum_{e \in \mathcal{E}_{h}^{i}}|e|\left[\Phi_{\mu}(e)\right]^{-2}\left\|\eta-\bar{\eta}_{T_{e}}\right\|_{L_{2}(e)}^{2}\right]^{1 / 2} \\
\times\left[\sum_{e \in \mathcal{E}_{h}^{i}} \frac{\left[\Phi_{\mu}(e)\right]^{2}}{|e|}\|\llbracket \boldsymbol{n} \cdot \boldsymbol{v} \rrbracket\|_{L_{2}(e)}^{2}\right]^{1 / 2} \\
\lesssim h|\eta|_{H^{1}(\Omega)}\|\boldsymbol{v}\|_{h}=h\|\nabla \eta\|_{L_{2}(\Omega)}\|\boldsymbol{v}\|_{h} .
\end{gathered}
$$

The estimate (5.27) follows from (5.28) and (5.30). 


\section{Convergence Analysis}

In this section, following the approach of Schatz for indefinite problems [17], we prove the well-posedness of the discrete problem (3.4) and estimate the discretization errors. We begin with three lemmas that provide estimates for the three terms on the right-hand side of (3.12).

Lemma 6.1. Let $\stackrel{\circ}{\boldsymbol{u}} \in H_{0}(\operatorname{curl} ; \Omega) \cap H\left(\operatorname{div}^{0} ; \Omega\right)$ be the solution of (1.3). It holds that

$$
\inf _{\boldsymbol{v} \in V_{h}}\|\stackrel{\circ}{\boldsymbol{u}}-\boldsymbol{v}\|_{h} \leq\left\|\stackrel{\circ}{\boldsymbol{u}}-\Pi_{h} \stackrel{\circ}{\boldsymbol{u}}\right\|_{h} \leq C_{\epsilon} h^{1-\epsilon}\|\boldsymbol{f}\|_{L_{2}(\Omega)}
$$

for any $\epsilon>0$.

Proof. According to (3.8), we have

$$
\begin{aligned}
\left\|\stackrel{\circ}{\boldsymbol{u}}-\Pi_{h} \stackrel{\circ}{\boldsymbol{u}}\right\|_{h}^{2}= & \left\|\nabla_{h} \times\left(\stackrel{\circ}{\boldsymbol{u}}-\Pi_{h} \stackrel{\circ}{\boldsymbol{u}}\right)\right\|_{L_{2}(\Omega)}^{2}+\left\|\stackrel{\circ}{\boldsymbol{u}}-\Pi_{h} \stackrel{\circ}{\boldsymbol{u}}\right\|_{L_{2}(\Omega)}^{2} \\
+\sum_{e \in \mathcal{E}_{h}} & \left.\frac{\left[\Phi_{\mu}(e)\right]^{2}}{|e|} \| \llbracket \boldsymbol{n} \times\left(\stackrel{\circ}{\boldsymbol{u}}-\Pi_{h} \stackrel{\circ}{\boldsymbol{u}}\right)\right] \|_{L_{2}(e)}^{2} \\
& +\sum_{e \in \mathcal{E}_{h}^{i}} \frac{\left[\Phi_{\mu}(e)\right]^{2}}{|e|}\left\|\llbracket \boldsymbol{n} \cdot\left(\stackrel{\circ}{\boldsymbol{u}}-\Pi_{h} \stackrel{\circ}{\boldsymbol{u}}\right)\right\| \|_{L_{2}(e)}^{2} .
\end{aligned}
$$

The second term on the right-hand side of (6.2) has been estimated in Lemma 5.1, and the third and fourth terms can be estimated using Lemma 5.2. Therefore it only remains to estimate the first term.

Observe that (2.2) implies

$$
\nabla_{h} \times\left(\Pi_{h} \stackrel{\circ}{\boldsymbol{u}}\right)=\Pi_{h}^{0}(\nabla \times \stackrel{\circ}{\boldsymbol{u}})
$$

where $\Pi_{h}^{0}$ is the orthogonal projection from $L_{2}(\Omega)$ onto the space of piecewise constant functions with respect to $\mathcal{T}_{h}$. It then follows from (1.6), (2.8), (6.3) and a standard interpolation error estimate [6, 5] that

$$
\begin{gathered}
\left\|\nabla_{h} \times\left(\stackrel{\circ}{\boldsymbol{u}}-\Pi_{h} \stackrel{\circ}{\boldsymbol{u}}\right)\right\|_{L_{2}(\Omega)}^{2}=\left\|\nabla \times \stackrel{\circ}{\boldsymbol{u}}-\Pi_{h}^{0}(\nabla \times \stackrel{\circ}{\boldsymbol{u}})\right\|_{L_{2}(\Omega)}^{2} \\
\lesssim h^{2}|\nabla \times \stackrel{\circ}{\boldsymbol{u}}|_{H^{1}(\Omega)}^{2} \lesssim h^{2}\|\boldsymbol{f}\|_{L_{2}(\Omega)}^{2} .
\end{gathered}
$$

The estimate (6.1) follows from (6.2), (6.4) and Lemmas 5.1 and 5.2

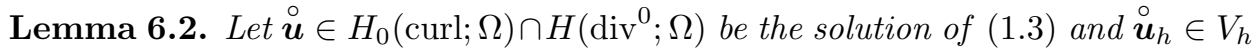
satisfy (3.4). It holds that

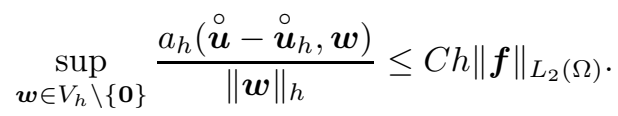

Proof. Let $\boldsymbol{w} \in V_{h}$ be arbitrary. We have, by (1.5), (3.2), (3.3), (3.5), and integration by parts,

$$
\begin{aligned}
a_{h}(\stackrel{\circ}{\boldsymbol{u}}, \boldsymbol{w}) & =\sum_{T \in \mathcal{T}_{h}} \int_{T}(\nabla \times \stackrel{\circ}{\boldsymbol{u}})(\nabla \times \boldsymbol{w}) d x-k^{2}(\stackrel{\circ}{\boldsymbol{u}}, \boldsymbol{w}) \\
& =(Q \boldsymbol{f}, \boldsymbol{w})+\sum_{e \in \mathcal{E}_{h}} \int_{e}(\nabla \times \stackrel{\circ}{\boldsymbol{u}}) \llbracket \boldsymbol{n} \times \boldsymbol{w} \rrbracket d s
\end{aligned}
$$


Subtracting (3.4) from (6.6), we find

$$
a_{h}\left(\stackrel{\circ}{\boldsymbol{u}}-\stackrel{\circ}{\boldsymbol{u}}_{h}, \boldsymbol{w}\right)=(Q \boldsymbol{f}-\boldsymbol{f}, \boldsymbol{w})+\sum_{e \in \mathcal{E}_{h}} \int_{e}(\nabla \times \stackrel{\circ}{\boldsymbol{u}}) \llbracket \boldsymbol{n} \times \boldsymbol{w} \rrbracket d s .
$$

The first term on the right-hand side of (6.7) can be estimated using Lemma 5.4

$$
\begin{aligned}
(Q \boldsymbol{f}-\boldsymbol{f}, \boldsymbol{w}) & =(\boldsymbol{f}, Q \boldsymbol{w}-\boldsymbol{w}) \\
& \leq\|\boldsymbol{f}\|_{L_{2}(\Omega)}\|Q \boldsymbol{w}-\boldsymbol{w}\|_{L_{2}(\Omega)} \lesssim h\|\boldsymbol{f}\|_{L_{2}(\Omega)}\|\boldsymbol{w}\|_{h} .
\end{aligned}
$$

The second term can also be estimated by arguments similar to those in the proof of Lemma 5.4. Since $\boldsymbol{w}$ is continuous at the midpoints, we can write

$$
\sum_{e \in \mathcal{E}_{h}} \int_{e}(\nabla \times \stackrel{\circ}{\boldsymbol{u}}) \llbracket \boldsymbol{n} \times \boldsymbol{w} \rrbracket d s=\sum_{e \in \mathcal{E}_{h}} \int_{e}\left(\nabla \times \stackrel{\circ}{\boldsymbol{u}}-\overline{(\nabla \times \stackrel{\circ}{\boldsymbol{u}})_{T_{e}}}\right) \llbracket \boldsymbol{n} \times \boldsymbol{w} \rrbracket d s,
$$

where $\overline{(\nabla \times \stackrel{\circ}{ })_{T_{e}}}$ is the mean of $\nabla \times \stackrel{\circ}{\boldsymbol{u}}$ on $T_{e}$, one of the triangles in $\mathcal{T}_{h}$ that has $e$ as an edge. It then follows from (1.6), (3.8), the Cauchy-Schwarz inequality, and Lemma 5.3 that

$$
\begin{aligned}
& \sum_{e \in \mathcal{E}_{h}} \int_{e}(\nabla \times \stackrel{\circ}{u}) \llbracket \boldsymbol{n} \times \boldsymbol{w} \rrbracket d s \\
& \leq\left[\sum_{e \in \mathcal{E}_{h}}|e|\left[\Phi_{\mu}(e)\right]^{-2}\left\|\nabla \times \stackrel{\circ}{\boldsymbol{u}}-\overline{(\nabla \times \stackrel{\circ}{u})_{T_{e}}}\right\|_{L_{2}(e)}^{2}\right]^{1 / 2} \\
& \times\left[\sum_{e \in \mathcal{E}_{h}} \frac{\left[\Phi_{\mu}(e)\right]^{2}}{|e|}\|\llbracket \boldsymbol{n} \times \boldsymbol{w} \rrbracket\|_{L_{2}(e)}^{2}\right]^{1 / 2} \\
& \lesssim h \mid \nabla \times \stackrel{\circ}{\left.\right|_{H^{1}(\Omega)}}\|\boldsymbol{w}\|_{h} \lesssim h\|\boldsymbol{f}\|_{L_{2}(\Omega)}\|\boldsymbol{w}\|_{h},
\end{aligned}
$$

which together with (6.7) and (6.8) completes the proof.

Remark 6.3. The analysis carried out in Lemma 6.2 explains why the straightforward discretization,

$$
\left(\nabla_{h} \times \stackrel{\circ}{u}_{h}, \nabla_{h} \times \boldsymbol{v}\right)-k^{2}\left(\stackrel{\circ}{u}_{h}, \boldsymbol{v}\right)=(\boldsymbol{f}, \boldsymbol{v}) \quad \forall \boldsymbol{v} \in V_{h}
$$

without the consistency term fails to converge (cf. [15], page 200 and Table 7.2 below). Indeed, we can see from (5.29) and (6.7) that both $\llbracket \boldsymbol{n} \cdot \boldsymbol{w} \rrbracket$ and $\llbracket \boldsymbol{n} \times \boldsymbol{w} \rrbracket$ appear naturally in the consistency analysis of the method. For the Poisson problem the jumps of nonconforming $P_{1}$ functions can be controlled by the piecewise $H^{1}$ norm using the continuity at the midpoints. But for the Maxwell equations the piecewise $H$ (curl) norm is not strong enough to control $\llbracket \boldsymbol{n} \cdot \boldsymbol{w} \rrbracket$ and $\llbracket \boldsymbol{n} \times \boldsymbol{w} \rrbracket$, even with the continuity of $\boldsymbol{w}$ at the midpoints.

Remark 6.4. Even though our analysis relies on the fact that $\|\cdot\|_{h}$ defined in (3.8) includes the normal jumps, it is still possible that a discretization without the normal jumps would converge. For example, we can replace the bilinear form $a_{h}(\cdot, \cdot)$ in $(3.4)$ by the bilinear form

$$
\begin{aligned}
\tilde{a}_{h}(\boldsymbol{w}, \boldsymbol{v})=\left(\nabla_{h}\right. & \left.\times \boldsymbol{w}, \nabla_{h} \times \boldsymbol{v}\right)-k^{2}(\boldsymbol{w}, \boldsymbol{v}) \\
& +\sum_{e \in \mathcal{E}_{h}} \frac{\left[\Phi_{\mu}(e)\right]^{2}}{|e|} \int_{e} \llbracket \boldsymbol{n} \times \boldsymbol{w} \rrbracket \llbracket \boldsymbol{n} \times \boldsymbol{v} \rrbracket d s
\end{aligned}
$$


The resulting scheme in fact converges. Numerical results can be found in Table 7.3 below, where the norm $\|\cdot\|_{h}$ is defined by

$$
\|\boldsymbol{v}\|_{h}^{2}=\left\|\nabla_{h} \times \boldsymbol{v}\right\|_{L_{2}(\Omega)}^{2}+\|\boldsymbol{v}\|_{L_{2}(\Omega)}^{2}+\sum_{e \in \mathcal{E}_{h}} \frac{\left[\Phi_{\mu}(e)\right]^{2}}{|e|}\|\llbracket \boldsymbol{n} \times \boldsymbol{v} \rrbracket\|_{L_{2}(e)}^{2} .
$$

But we will not analyze this scheme in the current paper.

Lemma 6.5. Let $\stackrel{\circ}{\boldsymbol{u}} \in H_{0}(\operatorname{curl} ; \Omega) \cap H\left(\operatorname{div}^{0} ; \Omega\right)$ be the solution of (1.3) and $\stackrel{\circ}{\boldsymbol{u}}_{h} \in V_{h}$ satisfy (3.4). It holds that

$$
\left\|\stackrel{\circ}{\boldsymbol{u}}-\stackrel{\circ}{\boldsymbol{u}}_{h}\right\|_{L_{2}(\Omega)} \leq C_{\epsilon}\left(h^{2-\epsilon}\|\boldsymbol{f}\|_{L_{2}(\Omega)}+h^{1-\epsilon}\left\|\stackrel{\circ}{\boldsymbol{u}}-\stackrel{\circ}{\boldsymbol{u}}_{h}\right\|_{h}\right)
$$

for any $\epsilon>0$.

Proof. We will establish (6.12) by a duality argument.

Let $\stackrel{\circ}{\boldsymbol{z}} \in H_{0}(\operatorname{curl} ; \Omega) \cap H\left(\operatorname{div}^{0} ; \Omega\right)$ satisfy the RTHM equations

$$
(\nabla \times \boldsymbol{v}, \nabla \times \stackrel{\circ}{\boldsymbol{z}})-k^{2}(\boldsymbol{v}, \stackrel{\circ}{\boldsymbol{z}})=\left(\boldsymbol{v}, \stackrel{\circ}{\boldsymbol{u}}-\stackrel{\circ}{\boldsymbol{u}}_{h}\right) \quad \forall \boldsymbol{v} \in H_{0}(\operatorname{curl} ; \Omega) \cap H\left(\operatorname{div}^{0} ; \Omega\right),
$$

which can also be written as

$$
a_{h}(\boldsymbol{v}, \stackrel{\circ}{\boldsymbol{z}})=\left(\boldsymbol{v}, \stackrel{\circ}{\boldsymbol{u}}-\stackrel{\circ}{\boldsymbol{u}}_{h}\right) \quad \forall \boldsymbol{v} \in H_{0}(\operatorname{curl} ; \Omega) \cap H\left(\operatorname{div}^{0} ; \Omega\right) .
$$

The strong form of (6.13) is

$$
\nabla \times(\nabla \times \stackrel{\circ}{\boldsymbol{z}})-k^{2} \stackrel{\circ}{\boldsymbol{z}}=Q\left(\stackrel{\circ}{\boldsymbol{u}}-\stackrel{\circ}{\boldsymbol{u}}_{h}\right)
$$

and we have the following analog of (1.6):

$$
|\nabla \times \stackrel{\circ}{\boldsymbol{z}}|_{H^{1}(\Omega)} \lesssim\left\|\stackrel{\circ}{\boldsymbol{u}}-\stackrel{\circ}{\boldsymbol{u}}_{h}\right\|_{L_{2}(\Omega)}
$$

From (6.13) we have

$$
\left(\stackrel{\circ}{\boldsymbol{u}}, \stackrel{\circ}{\boldsymbol{u}}-\stackrel{\circ}{\boldsymbol{u}}_{h}\right)=a_{h}(\stackrel{\circ}{\boldsymbol{u}}, \stackrel{\circ}{\boldsymbol{z}}) .
$$

On the other hand, it follows from (6.14) and integration by parts that the following analog of (6.6) holds:

$$
a_{h}\left(\stackrel{\circ}{\boldsymbol{u}}_{h}, \stackrel{\circ}{\boldsymbol{z}}\right)=\left(\stackrel{\circ}{\boldsymbol{u}}_{h}, Q\left(\stackrel{\circ}{\boldsymbol{u}}-\stackrel{\circ}{\boldsymbol{u}}_{h}\right)\right)+\sum_{e \in \mathcal{E}_{h}} \int_{e}(\nabla \times \stackrel{\circ}{\mathbf{z}}) \llbracket \boldsymbol{n} \times \stackrel{\circ}{\boldsymbol{u}}_{h} \rrbracket d s .
$$

Combining (6.16) and (6.17), we find

$$
\begin{aligned}
& \left\|\stackrel{\circ}{\boldsymbol{u}}-\stackrel{\circ}{\boldsymbol{u}}_{h}\right\|_{L_{2}(\Omega)}^{2}=\left(\stackrel{\circ}{\boldsymbol{u}}, \stackrel{\circ}{\boldsymbol{u}}-\stackrel{\circ}{\boldsymbol{u}}_{h}\right)-\left(\stackrel{\circ}{\boldsymbol{u}}_{h}, \stackrel{\circ}{\boldsymbol{u}}-\stackrel{\circ}{\boldsymbol{u}}_{h}\right) \\
& =a_{h}\left(\stackrel{\circ}{\boldsymbol{u}}-\stackrel{\circ}{\boldsymbol{u}}_{h}, \stackrel{\circ}{\boldsymbol{z}}\right)-\left(\stackrel{\circ}{\boldsymbol{u}}_{h},(I-Q)\left(\stackrel{\circ}{\boldsymbol{u}}-\stackrel{\circ}{\boldsymbol{u}}_{h}\right)\right) \\
& +\sum_{e \in \mathcal{E}_{h}} \int_{e}(\nabla \times \stackrel{\circ}{\boldsymbol{z}}) \llbracket \boldsymbol{n} \times \stackrel{\circ}{\boldsymbol{u}}_{h} \rrbracket d s .
\end{aligned}
$$

We will estimate the three terms on the right-hand side of (6.18) separately.

We can write the first term as

$$
a_{h}\left(\stackrel{\circ}{\boldsymbol{u}}-\stackrel{\circ}{\boldsymbol{u}}_{h}, \stackrel{\circ}{\boldsymbol{z}}\right)=a_{h}\left(\stackrel{\circ}{\boldsymbol{u}}-\stackrel{\circ}{\boldsymbol{u}}_{h}, \stackrel{\circ}{\boldsymbol{z}}-\Pi_{h} \stackrel{\circ}{\boldsymbol{z}}\right)+a_{h}\left(\stackrel{\circ}{\boldsymbol{u}}-\stackrel{\circ}{\boldsymbol{u}}_{h}, \Pi_{h} \stackrel{\circ}{\boldsymbol{z}}\right),
$$

and from (3.10) and Lemma 6.1 (applied to $\stackrel{\circ}{z}$ ) we immediately have the following estimate:

$$
\begin{aligned}
a_{h}\left(\stackrel{\circ}{\boldsymbol{u}}-\stackrel{\circ}{\boldsymbol{u}}_{h}, \stackrel{\circ}{\boldsymbol{z}}-\Pi_{h} \stackrel{\circ}{\boldsymbol{z}}\right) & \leq C\left\|\stackrel{\circ}{\boldsymbol{u}}-\stackrel{\circ}{\boldsymbol{u}}_{h}\right\|_{h}\left\|\stackrel{\circ}{\boldsymbol{z}}-\Pi_{h} \stackrel{\circ}{\boldsymbol{z}}\right\|_{h} \\
& \leq C_{\epsilon} h^{1-\epsilon}\left\|\stackrel{\circ}{\boldsymbol{u}}-\stackrel{\circ}{\boldsymbol{u}}_{h}\right\|_{h}\left\|\stackrel{\circ}{\boldsymbol{u}}-\stackrel{\circ}{\boldsymbol{u}}_{h}\right\|_{L_{2}(\Omega)} .
\end{aligned}
$$


From (6.7) we can rewrite the second term on the right-hand side of (6.19) as

$$
a_{h}\left(\stackrel{\circ}{\boldsymbol{u}}-\stackrel{\circ}{\boldsymbol{u}}_{h}, \Pi_{h} \stackrel{\circ}{\boldsymbol{z}}\right)=\left(Q \boldsymbol{f}-\boldsymbol{f}, \Pi_{h} \stackrel{\circ}{\boldsymbol{z}}\right)+\sum_{e \in \mathcal{E}_{h}} \int_{e}(\nabla \times \stackrel{\circ}{\boldsymbol{u}}) \llbracket \boldsymbol{n} \times \Pi_{h} \stackrel{\circ}{\boldsymbol{z}} \rrbracket d s .
$$

Since $\stackrel{\circ}{\boldsymbol{z}} \in H\left(\operatorname{div}^{0} ; \Omega\right)$, we have $Q \stackrel{\circ}{\boldsymbol{z}}=\stackrel{\circ}{\boldsymbol{z}}$ and, by Lemma 5.1 (applied to $\stackrel{\circ}{\boldsymbol{z}}$ ),

$$
\begin{aligned}
(Q \boldsymbol{f}-\boldsymbol{f} & \left., \Pi_{h} \stackrel{\circ}{\boldsymbol{z}}\right)=\left(\boldsymbol{f}, Q \Pi_{h} \stackrel{\circ}{\boldsymbol{z}}-\Pi_{h} \stackrel{\circ}{\boldsymbol{z}}\right) \\
& =\left(\boldsymbol{f}, Q\left(\Pi_{h} \stackrel{\circ}{\boldsymbol{z}}-\stackrel{\circ}{\boldsymbol{z}}\right)-\left(\Pi_{h} \stackrel{\circ}{\boldsymbol{z}}-\stackrel{\circ}{\boldsymbol{z}}\right)\right) \\
& \leq\|\boldsymbol{f}\|_{L_{2}(\Omega)}\left\|\Pi_{h} \stackrel{\circ}{\boldsymbol{z}}-\stackrel{\circ}{\boldsymbol{z}}\right\|_{L_{2}(\Omega)} \\
& \leq\|\boldsymbol{f}\|_{L_{2}(\Omega)}\left(C_{\epsilon} h^{2-\epsilon}\left\|\stackrel{\circ}{\boldsymbol{u}}-\stackrel{\circ}{\boldsymbol{u}}_{h}\right\|_{L_{2}(\Omega)}\right) .
\end{aligned}
$$

We can rewrite the second term on the right-hand side of (6.21) using the notation introduced in (5.26):

$$
\begin{gathered}
\sum_{e \in \mathcal{E}_{h}} \int_{e}(\nabla \times \stackrel{\circ}{\boldsymbol{u}}) \llbracket \boldsymbol{n} \times \Pi_{h} \stackrel{\circ}{\boldsymbol{z}} \rrbracket d s=\sum_{e \in \mathcal{E}_{h}} \int_{e}\left(\nabla \times \stackrel{\circ}{\boldsymbol{u}}-\overline{(\nabla \times \stackrel{\circ}{\boldsymbol{u}})_{T_{e}}}\right) \llbracket \boldsymbol{n} \times \Pi_{h} \stackrel{\circ}{\boldsymbol{z}} \rrbracket d s \\
=\sum_{e \in \mathcal{E}_{h}} \int_{e}\left(\nabla \times \stackrel{\circ}{\boldsymbol{u}}-{\overline{(\nabla \times \stackrel{\circ}{\mathbf{u}})_{T_{e}}}}\right) \llbracket \boldsymbol{n} \times\left(\Pi_{h} \stackrel{\circ}{\boldsymbol{z}}-\stackrel{\circ}{\boldsymbol{z}}\right) \rrbracket d s
\end{gathered}
$$

since $\boldsymbol{n} \times \Pi_{h} \stackrel{\circ}{\boldsymbol{z}}$ is continuous at the midpoint of any edge $e \in \mathcal{E}_{h}^{i}$ and vanishes

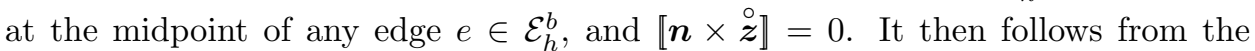
Cauchy-Schwarz inequality, (1.6), Lemma $[5.2$ (applied to $\stackrel{\circ}{z}$ ), and Lemma $[5.3$ that

$$
\begin{aligned}
& \sum_{e \in \mathcal{E}_{h}} \int_{e}(\nabla \times \stackrel{\circ}{\boldsymbol{u}}) \llbracket \boldsymbol{n} \times \Pi_{h} \stackrel{\circ}{\boldsymbol{z}} \rrbracket d s \\
& \leq\left[\sum_{e \in \mathcal{E}_{h}}|e|\left[\Phi_{\mu}(e)\right]^{-2} \| \nabla \times \stackrel{\circ}{\boldsymbol{u}}-{\overline{(\nabla \times \stackrel{\circ}{u})_{T_{e}}}}_{L_{L_{2}(e)}}\right]^{1 / 2} \\
& \times\left[\sum_{e \in \mathcal{E}_{h}} \frac{\left[\Phi_{\mu}(e)\right]^{2}}{|e|} \|\left[\Pi_{h} \stackrel{\circ}{\boldsymbol{z}}-\stackrel{\circ}{\boldsymbol{z}} \rrbracket \|_{L_{2}(e)}^{2}\right]^{1 / 2}\right. \\
& \leq C_{\epsilon}\left(h \mid \nabla \times \stackrel{\circ}{\left.\right|_{H^{1}(\Omega)}}\right)\left(h^{1-\epsilon} \| \stackrel{\circ}{\boldsymbol{u}}-{\stackrel{\circ}{\boldsymbol{u}_{h}}}_{\|_{L_{2}(\Omega)}}\right) \\
& \leq C_{\epsilon} h^{2-\epsilon}\|\boldsymbol{f}\|_{L_{2}(\Omega)}\left\|\stackrel{\circ}{\boldsymbol{u}}-{\stackrel{\circ}{\boldsymbol{u}_{h}}}\right\|_{L_{2}(\Omega)} .
\end{aligned}
$$

Combining (6.19) -6.23 we find

$$
a_{h}\left(\stackrel{\circ}{\boldsymbol{u}}-\stackrel{\circ}{u}_{h}, \stackrel{\circ}{\boldsymbol{z}}\right) \leq C_{\epsilon}\left(h^{2-\epsilon}\|\boldsymbol{f}\|_{L_{2}(\Omega)}+h^{1-\epsilon}\left\|\stackrel{\circ}{\boldsymbol{u}}-\stackrel{\circ}{\boldsymbol{u}}_{h}\right\|_{h}\right) \| \stackrel{\circ}{\boldsymbol{u}}-{\stackrel{\circ}{\boldsymbol{u}_{h}}}_{\|_{L_{2}(\Omega)}}
$$

Next we estimate the second term on the right-hand side of (6.18) by Lemma 5.4.

$$
\begin{aligned}
-\left(\stackrel{\circ}{\boldsymbol{u}}_{h},(I-Q)\left(\stackrel{\circ}{\boldsymbol{u}}-{\stackrel{\circ}{\boldsymbol{u}_{h}}}_{h}\right)\right. & =\left(\stackrel{\circ}{\boldsymbol{u}}-\stackrel{\circ}{\boldsymbol{u}}_{h},(I-Q)\left(\stackrel{\circ}{\boldsymbol{u}}-{\stackrel{\circ}{\boldsymbol{u}_{h}}}_{h}\right)\right. \\
& \leq C\left\|\stackrel{\circ}{\boldsymbol{u}}-\stackrel{\circ}{\boldsymbol{u}}_{h}\right\|_{L_{2}(\Omega)}\left(h\left\|\boldsymbol{u}-\stackrel{\circ}{\boldsymbol{u}}_{h}\right\|_{h}\right),
\end{aligned}
$$

where we have used the fact that $(I-Q) \stackrel{\circ}{\boldsymbol{u}}=\mathbf{0}$.

Finally we estimate the third term on the right-hand side of (6.18). Since $\boldsymbol{n} \times{\stackrel{\circ}{\boldsymbol{u}_{h}}}$ is continuous at the midpoints of the interior edges and vanishes at the midpoints 
of the boundary edges, and $\llbracket \boldsymbol{n} \times \stackrel{\circ}{\boldsymbol{u}} \rrbracket=0$, we can write, following the notation in (5.26),

$$
\begin{aligned}
\sum_{e \in \mathcal{E}_{h}} \int_{e}(\nabla \times \stackrel{\circ}{\boldsymbol{z}}) \llbracket \boldsymbol{n} \times \stackrel{\circ}{\boldsymbol{u}}_{h} \rrbracket d s \\
=\sum_{e \in \mathcal{E}_{h}} \int_{e}\left(\nabla \times \stackrel{\circ}{\boldsymbol{z}}-\overline{(\nabla \times \stackrel{\circ}{\boldsymbol{z}})_{T_{e}}}\right) \llbracket \boldsymbol{n} \times \stackrel{\circ}{\boldsymbol{u}}_{h} \rrbracket d s \\
=\sum_{e \in \mathcal{E}_{h}} \int_{e}\left(\nabla \times \stackrel{\circ}{\boldsymbol{z}}-\overline{(\nabla \times \stackrel{\circ}{\boldsymbol{z}})_{T_{e}}}\right) \llbracket \boldsymbol{n} \times\left(\stackrel{\circ}{\boldsymbol{u}}_{h}-\stackrel{\circ}{\boldsymbol{u}}\right) \rrbracket d s
\end{aligned}
$$

Using the Cauchy-Schwarz inequality, (3.8), (6.15) and Lemma 5.3 (applied to $\stackrel{\circ}{\boldsymbol{z}}$ ), we obtain

$$
\begin{aligned}
& \sum_{e \in \mathcal{E}_{h}} \int_{e}(\nabla \times \stackrel{\circ}{\boldsymbol{z}}) \llbracket \boldsymbol{n} \times \stackrel{\circ}{\boldsymbol{u}}_{h} \rrbracket d s \\
& \leq\left[\sum_{e \in \mathcal{E}_{h}}|e|\left[\Phi_{\mu}(e)\right]^{-2}\left\|\nabla \times \stackrel{\circ}{\boldsymbol{z}}-\overline{(\nabla \times \stackrel{\circ}{\boldsymbol{z}})_{T_{e}}}\right\|_{L_{2}(e)}^{2}\right]^{1 / 2} \\
& \times\left[\sum_{e \in \mathcal{E}_{h}} \frac{\left[\Phi_{\mu}(e)\right]^{2}}{|e|}\left\|\llbracket \stackrel{\circ}{\boldsymbol{u}}_{h}-\stackrel{\circ}{u} \rrbracket\right\|_{L_{2}(e)}^{2}\right]^{1 / 2} \\
& \leq C h|\nabla \times \stackrel{\circ}{\boldsymbol{z}}|_{H^{1}(\Omega)}\left\|\stackrel{\circ}{\boldsymbol{u}}_{h}-\stackrel{\circ}{\boldsymbol{u}}\right\|_{h} \\
& \leq C h\left\|\stackrel{\circ}{\boldsymbol{u}}-{\stackrel{\circ}{\boldsymbol{u}_{h}}}_{h}\right\|_{L_{2}(\Omega)}\left\|\stackrel{\circ}{\boldsymbol{u}}-\stackrel{\circ}{\boldsymbol{u}}_{h}\right\|_{h} .
\end{aligned}
$$

The estimate (6.12) follows from (6.18) and (6.24) $-(6.26)$.

We are now ready to prove the main result of this paper.

Theorem 6.6. There exists a positive number $h_{*}$ such that the discrete problem (3.4) is uniquely solvable for all $h \leq h_{*}$, in which case the following discretization error estimates are valid:

$$
\begin{aligned}
& \left\|\stackrel{\circ}{\boldsymbol{u}}-\stackrel{\circ}{\boldsymbol{u}}_{h}\right\|_{h} \leq C_{\epsilon} h^{1-\epsilon}\|\boldsymbol{f}\|_{L_{2}(\Omega)} \quad \text { for any } \quad \epsilon>0, \\
& \left\|\stackrel{\circ}{\boldsymbol{u}}-{\stackrel{\circ}{\boldsymbol{u}_{h}}}_{\|}\right\|_{L_{2}(\Omega)} \leq C_{\epsilon} h^{2-\epsilon}\|\boldsymbol{f}\|_{L_{2}(\Omega)} \quad \text { for any } \epsilon>0 \text {. }
\end{aligned}
$$

Proof. Assuming $\stackrel{\circ}{u}_{h}$ satisfies (3.4), it follows from (3.12) and Lemmas 6.1, 6.2 and 6.5 that

$$
\left\|\stackrel{\circ}{\boldsymbol{u}}-{\stackrel{\circ}{\boldsymbol{u}_{h}}}_{h}\right\|_{h} \leq C_{\epsilon} h^{1-\epsilon}\left(\|\boldsymbol{f}\|_{L_{2}(\Omega)}+\| \stackrel{\circ}{\boldsymbol{u}}-{\stackrel{\circ}{\boldsymbol{u}_{h}}}_{\|_{h}}\right) \quad \text { for any } \epsilon>0 .
$$

By choosing an $\epsilon_{*}>0$, we deduce from (6.29) that, for $h \leq h_{*}=1 /\left(2 C_{\epsilon_{*}}\right)^{1 /\left(1-\epsilon_{*}\right)}$,

$$
\begin{aligned}
\left\|\stackrel{\circ}{\boldsymbol{u}}-\stackrel{\circ}{\boldsymbol{u}}_{h}\right\|_{h} & \leq C_{\epsilon_{*}} h^{1-\epsilon_{*}}\left(\|\boldsymbol{f}\|_{L_{2}(\Omega)}+\left\|\stackrel{\circ}{\boldsymbol{u}}-\stackrel{\circ}{\boldsymbol{u}}_{h}\right\|_{h}\right) \\
& \leq C_{\epsilon_{*}} h^{1-\epsilon_{*}}\|\boldsymbol{f}\|_{L_{2}(\Omega)}+C_{\epsilon_{*}} h_{*}^{1-\epsilon_{*}}\left\|\stackrel{\circ}{\boldsymbol{u}}-\stackrel{\circ}{\boldsymbol{u}}_{h}\right\|_{h} \\
& \leq C_{\epsilon_{*}} h^{1-\epsilon_{*}}\|\boldsymbol{f}\|_{L_{2}(\Omega)}+\frac{1}{2}\left\|\stackrel{\circ}{\boldsymbol{u}}-\stackrel{\circ}{\boldsymbol{u}}_{h}\right\|_{h},
\end{aligned}
$$

and hence

$$
\left\|\stackrel{\circ}{\boldsymbol{u}}-\stackrel{\circ}{u}_{h}\right\|_{h} \leq 2 C_{\epsilon_{*}} h^{1-\epsilon_{*}}\|\boldsymbol{f}\|_{L_{2}(\Omega)} .
$$


Therefore, any solution $\stackrel{\circ}{\boldsymbol{z}}_{h} \in V_{h}$ of the homogeneous discrete problem

$$
a_{h}\left(\stackrel{\circ}{\boldsymbol{z}}_{h}, \boldsymbol{v}\right)=0 \quad \forall \boldsymbol{v} \in V_{h},
$$

which corresponds to the special case where $\boldsymbol{f}=\mathbf{0}=\stackrel{\circ}{\boldsymbol{z}}$, will satisfy the following special case of $(\underline{6.30})$ :

$$
\left\|\stackrel{\circ}{\boldsymbol{z}}_{h}\right\|_{h} \leq 0
$$

Hence the only solution of (6.31) is the trivial solution and the discrete problem (3.4) is uniquely solvable for $h \leq h_{*}$.

The energy error estimate (6.27) now follows from (6.30), and the $L_{2}$ error estimate 6.28) follows from Lemma 6.5 and 6.27).

\section{NumERICAL EXPERIMENTS}

In this section we report the results of several numerical experiments that corroborate our theoretical results. Besides the $L_{2}$ error $\left\|\stackrel{\circ}{\boldsymbol{u}}-\stackrel{\circ}{\boldsymbol{u}}_{h}\right\|_{L_{2}(\Omega)}$ and the energy error $\left\|\stackrel{\circ}{\boldsymbol{u}}-\stackrel{\circ}{\boldsymbol{u}}_{h}\right\|_{h}$, we have also computed the error in the $|\cdot|_{\text {curl }}$ semi-norm defined by

$$
|\boldsymbol{v}|_{\text {curl }}=\left\|\nabla_{h} \times \boldsymbol{v}\right\|_{L_{2}(\Omega)} .
$$

In the first experiment we check the convergence behavior of our numerical scheme (3.4) on the square $(0,0.5)^{2}$ with uniform meshes, where the exact solution is

$$
\stackrel{\circ}{\boldsymbol{u}}=[y(y-0.5) \sin (k y), x(x-0.5) \cos (k x)]
$$

for $k=0,1,10$ and 20 . The results are tabulated in Table 7.1. They agree with the estimates (6.27) and (6.28). Observe also that finer meshes are needed for computing satisfactory approximate solutions when the wave numbers become larger.

In the second experiment we check the behavior of the scheme (6.9) that does not have the consistency term. The results in Table 7.2 confirm that this scheme does not converge.

In the third experiment we test the convergence of the scheme (3.4) with the bilinear form $a_{h}(\cdot, \cdot)$ replaced by the bilinear form $\tilde{a}_{h}(\cdot, \cdot)$ defined in (6.10). Comparing Table 7.1 and Table 7.3 (where $\|\cdot\|_{h}$ is defined by (6.11)), we see that this scheme converges but it does not perform as well as the original scheme.

In the fourth experiment we apply our method to a problem on $(0,0.5)^{2}$ with $k=1$ and where the right-hand side $\boldsymbol{f}$ is given by

$$
\boldsymbol{f}(x, y)= \begin{cases}(0,1) & x>0.25, y>0.25 \\ (1,1) & x<0.25, y>0.25 \\ (-1,1) & x<0.25, y<0.25 \\ (1,0) & x>0.25, y<0.25\end{cases}
$$

The convergence rates in $L_{2}(\Omega)$, computed by subtracting the numerical solutions from consecutive grids $(h=1 / 10,1 / 20,1 / 40$ and $1 / 80)$, are reported in Table 7.4 . They agree with the estimate (6.28).

Since our numerical scheme is designed for the reduced time-harmonic Maxwell equation (1.5), its performance should not be affected by the addition to the righthand side of a gradient term $\nabla G$ where $G \in H_{0}^{1}(\Omega)$. In the fifth experiment we add 
$\nabla G$, where

$$
G(x, y)=x y(x-0.5)(y-0.5) \sin (x+y),
$$

to the right-hand side

$$
\boldsymbol{f}=\nabla \times(\nabla \times \stackrel{\circ}{\boldsymbol{u}})-\stackrel{\circ}{\boldsymbol{u}}
$$

of the problem in the first experiment (with $k=1$ ) and compare their performance. The results are reported in Table 7.5, which demonstrate that indeed the performance of our method does not change if terms from $\nabla H_{0}^{1}(\Omega)$ are added to the right-hand side.

TABLE 7.1. Convergence of the scheme (3.4) on the square $(0,0.5)^{2}$

\begin{tabular}{|c|c|c|c|c|c|c|}
\hline$h$ & $\frac{\left\|\stackrel{\circ}{\boldsymbol{u}}-\stackrel{\circ}{\boldsymbol{u}}_{h}\right\|_{L_{2}(\Omega)}}{\|\stackrel{\circ}{\boldsymbol{u}}\|_{L_{2}(\Omega)}}$ & order & $\frac{\left\|\stackrel{\circ}{\boldsymbol{u}}-\stackrel{\circ}{\boldsymbol{u}}_{h}\right\|_{h}}{\|\stackrel{\circ}{\boldsymbol{u}}\|_{h}}$ & order & $\frac{\left|\stackrel{\circ}{\boldsymbol{u}}-\stackrel{\circ}{\boldsymbol{u}}_{h}\right|_{\text {curl }}}{|\stackrel{\circ}{\boldsymbol{u}}|_{\text {curl }}}$ & order \\
\hline \multicolumn{7}{|c|}{$k=0$} \\
\hline $1 / 10$ & $4.28 \mathrm{e}-02$ & - & $2.89 \mathrm{e}-01$ & - & $1.70 \mathrm{e}-01$ & - \\
\hline $1 / 20$ & $9.68 \mathrm{e}-03$ & 2.15 & $1.42 \mathrm{e}-01$ & 1.02 & $8.52 \mathrm{e}-02$ & 1.00 \\
\hline $1 / 40$ & $2.29 \mathrm{e}-03$ & 2.08 & $7.05 \mathrm{e}-02$ & 1.01 & $4.25 \mathrm{e}-02$ & 1.00 \\
\hline $1 / 80$ & $5.56 \mathrm{e}-04$ & 2.04 & $3.50 \mathrm{e}-02$ & 1.00 & $2.12 \mathrm{e}-02$ & 1.00 \\
\hline \multicolumn{7}{|c|}{$k=1$} \\
\hline $1 / 10$ & $3.83 \mathrm{e}-02$ & - & $2.70 \mathrm{e}-01$ & - & $1.64 \mathrm{e}-01$ & - \\
\hline $1 / 20$ & $8.64 \mathrm{e}-03$ & 2.15 & $1.33 \mathrm{e}-01$ & 1.02 & $8.14 \mathrm{e}-02$ & 1.00 \\
\hline $1 / 40$ & $2.04 \mathrm{e}-03$ & 2.08 & $6.60 \mathrm{e}-02$ & 1.01 & $4.09 \mathrm{e}-02$ & 1.00 \\
\hline $1 / 80$ & $4.95 \mathrm{e}-04$ & 2.04 & $3.29 \mathrm{e}-02$ & 1.01 & $2.05 \mathrm{e}-02$ & 1.00 \\
\hline \multicolumn{7}{|c|}{$k=10$} \\
\hline $1 / 10$ & $3.69 \mathrm{e}-01$ & - & $8.41 \mathrm{e}-01$ & - & $4.07 \mathrm{e}-01$ & - \\
\hline $1 / 20$ & $5.30 \mathrm{e}-02$ & 2.80 & $3.46 \mathrm{e}-01$ & 1.28 & $1.98 \mathrm{e}-01$ & 1.04 \\
\hline $1 / 40$ & $1.13 \mathrm{e}-02$ & 2.23 & $1.67 \mathrm{e}-01$ & 1.05 & $9.89 \mathrm{e}-02$ & 1.00 \\
\hline $1 / 80$ & $2.68 \mathrm{e}-03$ & 2.08 & $8.27 \mathrm{e}-02$ & 1.02 & $4.93 \mathrm{e}-02$ & 1.00 \\
\hline \multicolumn{7}{|c|}{$k=20$} \\
\hline $1 / 10$ & $1.20 \mathrm{e}+02$ & - & $1.14 \mathrm{e}+02$ & - & $3.28 \mathrm{e}+01$ & - \\
\hline $1 / 20$ & $2.59 \mathrm{e}-01$ & - & $5.95 \mathrm{e}-01$ & - & $3.51 \mathrm{e}-01$ & - \\
\hline $1 / 40$ & $6.49 \mathrm{e}-02$ & 1.99 & $2.59 \mathrm{e}-01$ & 1.20 & $1.59 \mathrm{e}-01$ & 1.14 \\
\hline $1 / 80$ & $1.85 \mathrm{e}-02$ & 1.81 & $1.25 \mathrm{e}-01$ & 1.05 & $7.60 \mathrm{e}-02$ & 1.06 \\
\hline
\end{tabular}
with uniform meshes and exact solution $\stackrel{\circ}{\boldsymbol{u}}$ given by (7.1)

TABLE 7.2. Lack of convergence of the scheme (6.9) on the square $(0,0.5)^{2}$ with uniform meshes and exact solution $\stackrel{\circ}{u}$ given by (17.1) with $k=1$

\begin{tabular}{|c|c|c|c|c|}
\hline$h$ & $\frac{\left\|\stackrel{\circ}{\boldsymbol{u}}-\stackrel{\circ}{\boldsymbol{u}}_{h}\right\|_{L_{2}(\Omega)}}{\|\stackrel{\circ}{\boldsymbol{u}}\|_{L_{2}(\Omega)}}$ & order & $\frac{\mid \stackrel{\circ}{\boldsymbol{u}-\left.\stackrel{\circ}{\boldsymbol{u}}_{h}\right|_{\text {curl }}}}{|\stackrel{\circ}{\boldsymbol{u}}|_{\text {curl }}}$ & order \\
\hline $1 / 10$ & $3.06 \mathrm{e}+01$ & - & $4.76 \mathrm{e}-01$ & - \\
$1 / 20$ & $3.07 \mathrm{e}+01$ & 0.00 & $4.61 \mathrm{e}-01$ & 0.04 \\
$1 / 40$ & $3.07 \mathrm{e}+01$ & 0.00 & $4.58 \mathrm{e}-01$ & 0.01 \\
$1 / 80$ & $3.07 \mathrm{e}+01$ & 0.00 & $4.56 \mathrm{e}-01$ & 0.00 \\
\hline
\end{tabular}


TABLE 7.3. Convergence of the scheme without the normal jumps on the square $(0,0.5)^{2}$ with uniform meshes and exact solution $\stackrel{\circ}{\boldsymbol{u}}$ given by (7.1) with $k=1$

\begin{tabular}{|c|c|c|c|c|c|c|}
\hline$h$ & $\frac{\left\|\stackrel{\circ}{\boldsymbol{u}}-\stackrel{\circ}{\boldsymbol{u}}_{h}\right\|_{L_{2}(\Omega)}}{\|\stackrel{\circ}{\boldsymbol{u}}\|_{L_{2}(\Omega)}}$ & order & $\frac{\left\|\stackrel{\circ}{\boldsymbol{u}}-\stackrel{\circ}{\boldsymbol{u}}_{h}\right\|_{h}}{\|\stackrel{\circ}{u}\|_{h}}$ & order & $\frac{\mid \stackrel{\circ}{\boldsymbol{u}-\left.\stackrel{\circ}{\boldsymbol{u}}_{h}\right|_{\text {curl }}}}{\left|\boldsymbol{\circ}^{\prime}\right|_{\text {curl }}}$ & order \\
\hline $1 / 10$ & $9.84 \mathrm{e}-02$ & - & $3.31 \mathrm{e}-01$ & - & $1.53 \mathrm{e}-01$ & - \\
\hline $1 / 20$ & $2.37 \mathrm{e}-02$ & 2.05 & $1.63 \mathrm{e}-01$ & 1.02 & $7.65 \mathrm{e}-02$ & 1.00 \\
\hline $1 / 40$ & $5.85 \mathrm{e}-03$ & 2.02 & $8.11 \mathrm{e}-02$ & 1.01 & $3.83 \mathrm{e}-02$ & 1.00 \\
\hline $1 / 80$ & $1.45 \mathrm{e}-03$ & 2.01 & $4.05 \mathrm{e}-02$ & 1.00 & $1.91 \mathrm{e}-02$ & 1.00 \\
\hline
\end{tabular}

TABLE 7.4. $L_{2}$ convergence of the scheme (3.4) on $(0,0.5)^{2}$ with $k=1$ for the piecewise constant right-hand side $\boldsymbol{f}$ given by (7.2)

\begin{tabular}{|c|c|c|c|}
\hline$\left\|\stackrel{\circ}{\boldsymbol{u}}_{h}-\stackrel{\circ}{\boldsymbol{u}}_{h / 2}\right\|_{L_{2}(\Omega)}$ & $5.4221 \mathrm{e}-04$ & $1.2497 \mathrm{e}-04$ & $2.9973 \mathrm{e}-05$ \\
\hline order & - & 2.12 & 2.06 \\
\hline
\end{tabular}

TABLE 7.5. Comparison of the performance of the scheme (3.4) on $(0,0.5)^{2}$ with $k=1$ for the right-hand sides $\boldsymbol{f}$ and $\boldsymbol{f}+\nabla G$, where $f$ is given by (7.4) and $G$ is given by (7.3)

\begin{tabular}{|c|c|c|}
\hline$h$ & rhs $=f$ & $\mathrm{rhs}=\boldsymbol{f}+\nabla G$ \\
\hline \multicolumn{3}{|c|}{$\left\|\stackrel{\circ}{\boldsymbol{u}}-\stackrel{\circ}{\boldsymbol{u}_{h}}\right\|_{L_{2}(\Omega)}$} \\
\hline $1 / 10$ & $\mathbf{0 . 0 0 0 8 7 2 6 7 6 2 0 4 5 4}$ & $\mathbf{0 . 0 0 0 8 7 2 5 6 4 4 8 4 9 0}$ \\
\hline $1 / 20$ & $\mathbf{0 . 0 0 0 1 9 6 6 6 3 4 0 2 1 0}$ & $\mathbf{0 . 0 0 0 1 9 6 6 5 4 2 1 7 0 2}$ \\
\hline $1 / 40$ & $\mathbf{0 . 0 0 0 0 4 6 5 8 3 2 8 2 9 9}$ & $\mathbf{0 . 0 0 0 0 4 6 5 8 3 2 0 6 8 3}$ \\
\hline $1 / 80$ & $\mathbf{0 . 0 0 0 0 1 1 3 3 1 8 8 2 5 4}$ & $\mathbf{0 . 0 0 0 0 1 1 3 3 2 1 2 6 5 3}$ \\
\hline \multicolumn{3}{|c|}{$\left\|\stackrel{\bullet}{\boldsymbol{u}}-\stackrel{\leftrightarrow}{\boldsymbol{u}}_{h}\right\|_{h}$} \\
\hline $1 / 10$ & 0.03990964283043 & 0.03991029627634 \\
\hline $1 / 20$ & $\mathbf{0 . 0 1 9 6 6 0 3 0 9 5 8 1 4 0}$ & $\mathbf{0 . 0 1 9 6 6 0 4 3 7 3 1 3 5 8}$ \\
\hline $1 / 40$ & $\mathbf{0 . 0 0 9 7 5 7 3 7 1 1 9 2 1 5}$ & $\mathbf{0 . 0 0 9 7 5 7 4 2 8 0 1 1 7 2}$ \\
\hline $1 / 80$ & $\mathbf{0 . 0 0 4 8 6 0 5 7 7 3 1 5 5 7}$ & $\mathbf{0 . 0 0 4 8 6 0 6 0 9 2 1 2 2 8}$ \\
\hline
\end{tabular}

In the final experiment we check the convergence behavior of our scheme on the $L$-shaped domain $(-0.5,0.5)^{2} \backslash[0,0.5]^{2}$ with corners $(0.5,0),(0,0),(0,0.5)$, $(-0.5,0.5),(-0.5,-0.5)$ and $(0.5,-0.5)$. We take $k=1$ and the exact solution is chosen to be

$$
\stackrel{\circ}{\boldsymbol{u}}=\nabla \times\left(r^{2 / 3} \cos \left(\frac{2}{3} \theta-\frac{\pi}{3}\right) \phi(r / 0.5)\right),
$$

where $(r, \theta)$ are the polar coordinates at the origin and the cut-off function is given by

$$
\phi(r)= \begin{cases}1 & r \leq 0.25 \\ -16(r-0.75)^{3} & \\ \times\left[5+15(r-0.75)+12(r-0.75)^{2}\right] & 0.25 \leq r \leq 0.75 \\ 0 & r \geq 0.75\end{cases}
$$


The meshes are graded around the re-entrant corner with the grading parameter equal to $1 / 3$. The results are tabulated in Table 7.6 and they agree with the estimates (6.27) and (6.28).

TABLE 7.6. Convergence of the scheme (3.4) with graded meshes on the $L$-shaped domain $(-0.5,0.5)^{2} \backslash[0,0.5]^{2}$ with $k=1$ and exact solution $\stackrel{\circ}{u}$ given by (7.5)

\begin{tabular}{|c|c|c|c|c|c|c|}
\hline$h$ & $\frac{\left\|\boldsymbol{u}-\boldsymbol{u}_{h}\right\|_{L_{2}(\Omega)}}{\|\stackrel{\circ}{\boldsymbol{u}}\|_{L_{2}(\Omega)}}$ & order & 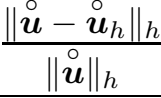 & order & 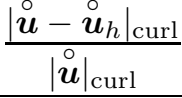 & order \\
\hline $1 / 4$ & $1.44 \mathrm{e}+02$ & - & $1.88 \mathrm{e}+01$ & - & $7.77 \mathrm{e}-00$ & - \\
\hline $1 / 8$ & $e+01$ & .92 & $7.39 \mathrm{e}-00$ & 1.35 & 4.41 & 0.82 \\
\hline $1 / 16$ & $3.35 \mathrm{e}-00$ & 3.50 & $2.24 \mathrm{e}-00$ & 1.73 & $7.87 \mathrm{e}-01$ & 2.49 \\
\hline $1 / 32$ & $6.88 \mathrm{e}-01$ & 2.28 & $1.09 \mathrm{e}-00$ & 1.03 & $4.34 \mathrm{e}-01$ & 0.86 \\
\hline $1 / 64$ & $1.51 \mathrm{e}-01$ & 2.18 & $5.51 \mathrm{e}-01$ & 0.99 & $2.34 \mathrm{e}-01$ & 0.90 \\
\hline
\end{tabular}

\section{CONCLUding REMARKS}

We have presented a nonconforming nodal finite element method for the twodimensional (reduced) time-harmonic Maxwell equations. Even though we have only discussed the perfectly conducting boundary condition, the scheme can be readily generalized to other boundary conditions (such as the impedance boundary condition [15]). However, the regularity of the solution and the error analysis of the scheme on graded meshes are then more involved and will be addressed elsewhere.

This method can be adapted for time-harmonic Maxwell equations in axisymmetric domains in three dimensions. It can also be extended to general threedimensional domains, where the construction of the locally-divergence basis is more complicated (cf. Section 9.3 of [10]).

Since our scheme computes approximations to vector fields in the space $H_{0}(\operatorname{curl} ; \Omega) \cap H\left(\operatorname{div}^{0} ; \Omega\right)$ that is responsible for the determination of Maxwell eigenvalues under the perfectly conducting boundary condition, it is also suitable for the computation of Maxwell eigenvalues. We have observed in preliminary numerical tests that the eigenvalues of the discrete operator converge to the Maxwell eigenvalues and there are no spurious eigenvalues. One can also take advantage of the theory of fast solvers for nodal nonconforming finite element methods to design fast solvers for the scheme (3.4), or the theory of error estimators for nodal nonconforming finite elements to develop adaptive versions of (3.4). These topics will be treated in our ongoing projects.

More importantly, our work in this paper shows that it is possible to design convergent nonconforming nodal finite element methods for electromagnetism. We expect that other new methods in this direction will be discovered.

\section{REFERENCES}

[1] Th. Apel. Anisotropic Finite Elements. Teubner, Stuttgart, 1999. MR1716824(2000k:65002)

[2] F. Assous, P. Ciarlet, Jr., and E. Sonnendrücker. Resolution of the Maxwell equation in a domain with reentrant corners. $M^{2} A N, 32: 359-389$, 1998. MR1627135 (2000b:78004)

[3] J.H. Bramble and S.R. Hilbert. Estimation of linear functionals on Sobolev spaces with applications to Fourier transforms and spline interpolation. SIAM J. Numer. Anal., 7:113-124, 1970. MR0263214 (41:7819) 
[4] S.C. Brenner and C. Carstensen. Finite Element Methods. In E. Stein, R. de Borst, and T.J.R. Hughes, editors, Encyclopedia of Computational Mechanics, pages 73-118. Wiley, Weinheim, 2004.

[5] S.C. Brenner and L.R. Scott. The Mathematical Theory of Finite Element Methods (Second Edition). Springer-Verlag, New York-Berlin-Heidelberg, 2002. MR.1894376 (2003a:65103)

[6] P.G. Ciarlet. The Finite Element Method for Elliptic Problems. North-Holland, Amsterdam, 1978. MR0520174 (58:25001)

[7] M. Costabel and M. Dauge. Singularities of electromagnetic fields in polyhedral domains. Arch. Ration. Mech. Anal., 151:221-276, 2000. MR.1753704(2002c:78005)

[8] M. Costabel and M. Dauge. Weighted regularization of Maxwell equations in polyhedral domains. Numer. Math., 93:239-277, 2002. MR.1941397 (2004a:78039)

[9] M. Crouzeix and P.-A. Raviart. Conforming and nonconforming finite element methods for solving the stationary Stokes equations I. RAIRO Anal. Numér., 7:33-75, 1973. MR0343661 $(49: 8401)$

[10] C. Cuvelier, A. Segal, and A.A. van Steenhoven. Finite Element Methods and Navier-Stokes Equations. D. Reidel Publishing Company, Dordrecht, 1986. MR0850259 (88g:65106)

[11] M. Dauge. Elliptic Boundary Value Problems on Corner Domains, Lecture Notes in Mathematics 1341. Springer-Verlag, Berlin-Heidelberg, 1988. MR0961439 (91a:35078)

[12] V. Girault and P.-A. Raviart. Finite Element Methods for Navier-Stokes Equations. Theory and Algorithms. Springer-Verlag, Berlin, 1986. MR0851383 (88b:65129)

[13] P. Grisvard. Elliptic Problems in Nonsmooth Domains. Pitman, Boston, 1985. MR0775683 (86m:35044)

[14] P. Houston, I. Perugia, A. Schneebeli, and D. Schötzau. Interior penalty method for the indefinite time-harmonic Maxwell equations. Numer. Math., 100:485-518, 2005. MR2194528 (2006k:65323)

[15] P. Monk. Finite Element Methods for Maxwell's Equations. Oxford University Press, New York, 2003. MR2059447(2005d:65003)

[16] I. Perugia, D. Schötzau, and P. Monk. Stabilized interior penalty methods for the timeharmonic Maxwell equations. Comput. Methods. Appl. Mech. Engrg., 191:4675-4697, 2002. MR1929626 (2003j:78058)

[17] A. Schatz. An observation concerning Ritz-Galerkin methods with indefinite bilinear forms. Math. Comp., 28:959-962, 1974. MR0373326 (51:9526)

[18] F. Thomasset. Implementation of Finite Element Methods for Navier-Stokes Equations. Springer-Verlag, New York-Berlin, 1981. MR0720192 (84k:76015)

Department of Mathematics, University of South Carolina, Columbia, South CarOLINA 29208

Current address: Department of Mathematics and Center for Computation and Technology, Louisiana State University, Baton Rouge, Louisiana 70803

E-mail address: brenner@math.1su.edu

Department of Mathematics, University of South Carolina, Columbia, South CarOLINA 29208

Current address: Department of Mathematical Sciences, Rensselaer Polytechnic Institute, Troy, New York 12180

E-mail address: lif@rpi.edu

Department of Mathematics, University of South Carolina, Columbia, South CarOLINA 29208

Current address: Department of Mathematics, Louisiana State University, Baton Rouge, Louisiana 70803

E-mail address: sung@math.lsu.edu 